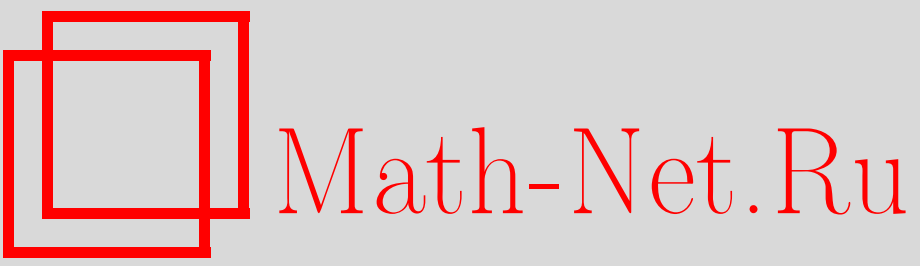

Ф. К. Клебанер, Р. Ш. Липцер, Когда стохастическая экспонента является мартингалом. Развитие метода Бенеша, Теория вероятн. и ее примен., 2013, том 58, выпуск 1, 53-80

DOI: https://doi.org/10.4213/tvp4494

Использование Общероссийского математического портала Math-Net.Ru подразумевает, что вы прочитали и согласны с пользовательским соглашением

http: //www . mathnet.ru/rus/agreement

Параметры загрузки :

IP: 35.173 .219 .12

26 апреля 2023 г., 07:34:07 


\title{
КОГДА СТОХАСТИЧЕСКАЯ ЭКСПОНЕНТА ЯВЛЯЕТСЯ МАРТИНГАЛОМ. РАЗВИТИЕ МЕТОДА БЕНЕША ${ }^{1)}$
}

\begin{abstract}
Стохастическая экспонента $\mathfrak{z}$ локального мартингала $M$ со скачками $\Delta M_{t}>-1$, т.е. $\mathfrak{z} t=1+\int_{0}^{t} \mathfrak{z}_{s-} d M_{s}$, является неотрицательным локальным мартингалом с $\mathbf{E}_{\mathfrak{z} t} \leqslant 1$. Если $\mathbf{E}_{\mathfrak{z}_{T}}=1$, то $\mathfrak{z}-$ мартингал на интервале $[0, T]$. Мартингальное свойство играет важную роль во многих приложениях. Поэтому естественные и легко проверяемые условия этого свойства представляют определенный интерес. В настоящей статье условие $\mathbf{E}_{\mathfrak{z}_{T}}=1$ проверяется при линейном росте параметров, участвующих в определении $M$, предложенные И.В. Гирсановым [10] и частично реализованные В. Бенешем [3]. Предлагаемый нами метод обобщает метод Бенеша без использования его технологии кусочно-постоянной аппроксимации. Предлагаемые условия эффективны в случаях, когда условия Новикова [30] и Казамаки [18] неприменимы. Они также эффективны в случае как марковских (возможно, взрывающихся), так и не марковских процессов, порождающих мартингалы $M$ со скачками. Наш подход отличается от недавно опубликованных подходов в статьях [5] и [29].
\end{abstract}

Ключевые слова и фразы: экспоненциальный мартингал, диффузионный процесс со скачкообразной компонентной, теорема Гирсанова, метод Бенеша.

1. Введение. Пусть $M=\left(M_{t}\right)_{t \in[0, T]}$ - мартингал (локальный мартингал) с траекториями из пространства Скорохода $\mathbb{D}$ и, значит, $M=M^{c}+M^{d}$, где $M^{c}$ и $M^{d}$ - непрерывный и чисто разрывный мартингалы. Обозначим $\Delta M_{t}:=M_{t}-M_{t-}$ и $\left\langle M^{c}\right\rangle_{t}$ соответственно процесс скачков и предсказуемую квадратическую вариацию непрерывного мартингала $M^{c}$. Если $\Delta M_{t}>-1, t>0$, то уравнение

$$
\mathfrak{z}_{t}=1+\int_{0}^{t} \mathfrak{z}_{s-} d M_{s}
$$

${ }^{*}$ School of Mathematical Sciences, Monash University, Australia; e-mail: fima.klebaner@sci.monash.edu.au

** Department of Electrical Engineering Systems, Tel Aviv University, Tel Aviv, Israel; e-mail: liptser@eng.tau.ac.il, rliptser@gmail.com

1) Работа выполнена при поддержке the Australian Research Council (гранты DP0881011 и DP0988483). 
имеет единственное решение (см., например, [9])

$$
\mathfrak{z}_{t}=\exp \left(M_{t}-\frac{1}{2}\left\langle M^{c}\right\rangle_{t}\right) \prod_{s=0}^{t}\left(1+\Delta M_{s}\right) e^{-\Delta M_{s}}, \quad t \geqslant 0,
$$

которое принято называть экспонентой Долеан-Дэд.

Хорошо известно, что $\mathfrak{z}$ - неотрицательный локальный мартингал и, значит, супермартингал с $\mathbf{E}_{\mathfrak{z} t} \leqslant 1$. Если же для некоторого $T>0$ выполняется условие

$$
\mathbf{E}_{\mathfrak{z}_{T}}=1,
$$

то $\mathfrak{z}=\left(\mathfrak{z}_{t}\right)_{t \in[0, T]}-$ мартингал, в частности, $\mathbf{E}_{\mathfrak{z} t} \equiv 1$ для любого $t \in[0, T]$. Свойство (1.3) эффективно используется в различных приложениях, где $\mathfrak{z}_{T}$ является производной Радона-Никодима, т.е. плотностью вероятностной меры относительно другой вероятностной меры в пространстве Скорохода. Случайная величина $\mathfrak{z}_{T}$ является одним из важных объектов в статистике случайных процессов (см. [26], [27]), в финансовой математике (см. [35], [36] и т.д.), при доказательстве существования слабых решений уравнений Ито (см. [33]) и других разнообразных приложениях.

Ниже приводится краткий обзор известных условий, гарантирующих равенство $\mathbf{E}_{\mathfrak{z}_{T}}=1$ при условии $M \equiv M^{c}$. В этом случае (1.3) имеет вид

$$
\mathbf{E} \exp \left(M_{T}^{c}-\frac{1}{2}\left\langle M^{c}\right\rangle_{T}\right)=1 .
$$

И. В. Гирсанов в своей классической статье [10] потребовал выполнения условия $\left\langle M^{c}\right\rangle_{T} \leqslant$ const. Это условие ослаблялось в разных вариантах, которые завершились условиями Новикова [30]

$$
\mathbf{E} \exp \left(\frac{1}{2}\left\langle M^{c}\right\rangle_{T}\right)<\infty,
$$

Казамаки [18]

$$
\sup _{t \in[0, T]} \mathbf{E} \exp \left(\frac{1}{2} M_{t}^{c}\right)<\infty
$$

и, наконец, Крылова [20]:

$$
\begin{gathered}
\lim _{\varepsilon \downarrow 0} \varepsilon \ln \mathbf{E} \exp \left([1-\varepsilon] \frac{1}{2}\left\langle M^{c}\right\rangle_{T}\right)<\infty, \\
\lim _{\varepsilon \downarrow 0} \varepsilon \ln \sup _{t \in[0, T]} \mathbf{E} \exp \left([1-\varepsilon] \frac{1}{2} M_{t}^{c}\right)<\infty .
\end{gathered}
$$

Следует отметить, что при любом $\varepsilon \in(0,1 / 2)$ можно подобрать мартингал $M^{c}$, для которого $\mathbf{E} e^{(1 / 2-\varepsilon)\left\langle M^{c}\right\rangle_{T}}<\infty$, но (1.3) не выполнено (см. [26, p. 224]). 
Рассмотрим простой пример. Пусть $B_{t}-$ броуновское движение и $M_{t}^{c}=2 \int_{0}^{t} B_{s} d B_{s}$. Тогда $\mathbf{E}_{\mathfrak{z}_{T}}<1$ при $T \geqslant T_{0}$, где $T_{0}$ определяется условием $\mathbf{E} \exp \left(B_{T_{0}}^{2}-T_{0}\right)=\infty$, т.е.

$$
\mathbf{E} \exp \left(2 \int_{0}^{T} B_{s} d B_{s}\right)=\mathbf{E} \exp \left(B_{T}^{2}-T\right)=\infty .
$$

Значит, условие Казамаки не выполнено и, как следствие, условие Новикова также не выполнено.

Все вышеупомянутые условия, обеспечивающие выполнение (1.3), формулируются в терминах $M^{c}$ и $\left\langle M^{c}\right\rangle$. Проверка этих условий требует весьма сложных, а подчас и неосуществимых вычислений.

Возникает естественный вопрос: как проверить (1.3) в рассматриваемом выше примере, если $T \geqslant T_{0}$ ? Оказывается, это можно сделать с помощью условия Бенеша [3].

Основная цель статьи - показать, что условие, называемое далее условием Бенеша, гарантирует выполнение (1.3) для достаточно общей структуры мартингала $M$ в (1.2).

Наряду с условием Бенеша мы используем условие равномерной интегрируемости, предложенное М. Хитсудой [11].

Отметим также, что предлагаемый метод проверки свойства $\mathbf{E}_{\mathfrak{z}_{T}}=1$ естественным образом совместим с методом проверки свойства $\mathbf{E}_{\mathfrak{z} \infty}=1$, предложенным в [14].

Другой подход в марковской модели можно найти в недавней статье $[5]$.

Для того чтобы сформулировать наш результат, предположим, что мартингал $M$ в (1.2) является частью структуры некоторого семимартингала $X$, участвующего в типичных прикладных моделях:

1) невзрывающийся марковский прочесс,

2) семимартингал, не являюшийся марковским прочессом,

3) марковский прочесс, не исключающий взрыьва.

Общий случай изучается в разделе 3 . В разделе 2 рассматривается случай непрерывного мартингала - более простой с технической точки зрения и допускающий применение метода Бенеша. В этом пункте мы показываем, как можно заменить доказательство Бенеша новым доказательством, обслуживающим самые разнообразные модели.

Эффективность основного результата проверяется на большом числе примеров (см. разделы 4-7).

Вспомогательный технический результат (обобщенная теорема Гирсанова) приводится в приложении А.

3 а м е ч а н и е 1 . Вопрос о том, является ли $\mathfrak{z}$ мартингалом, часто возникает в связи с другой проблемой. Пусть $\mu^{X}$ и $\mu^{Y}-$ вероятностные меры в пространстве Скорохода, являющиеся распределениями семимартингалов $X=\left(X_{t}\right)_{t \in[0, T]}$ и $Y=\left(Y_{t}\right)_{t \in[0, T]}$ соответственно. 
Возникает вопрос: когда $\mu^{X} \ll \mu^{Y}$ с производной Радона-Никодима $d \mu^{Y} / d \mu^{X}(X)=\mathfrak{z}_{T}$ ? Эти результаты можно найти в [10], [7], [12], [15], $[21],[23],[26$, гл. 7], [14] и более поздних статьях [33], [31], [37], [5], [29], [2] и т. д.

2. Условия Бенеша. Новый тип доказательства. В этом разделе излагаются основные идеи, имеющие отношение к вышеупомянутому примеру, не обслуживаемому условиями Новикова и Казамаки.

Мы рассматриваем два типа непрерывных мартингалов:

$$
M_{t}^{\prime}=\int_{0}^{t} \sigma\left(B_{s}\right) d B_{s} \quad \text { и } \quad M_{t}^{\prime \prime}=\int_{0}^{t} \sigma_{s}(B) d B_{s},
$$

где функции $\sigma(y)$ и $\sigma_{s}(y)$ аргументов $y \in \mathbf{R}_{\text {и }}\left(s, y_{[0, s]}\right) \in \mathbf{R}_{+} \times \mathbf{C}_{[0, \infty)}$ измеримы относительно соответствующих $\sigma$-алгебр и удовлетворяют условиям ««линейного роста»»:

$$
\begin{aligned}
\sigma^{2}(y) & \leqslant r\left[1+y^{2}\right], \\
\sigma^{2}\left(s, y_{[0, s]}\right) & \leqslant r\left[1+\sup _{s^{\prime} \leqslant s} y_{s^{\prime}}^{2}\right] .
\end{aligned}
$$

Как хорошо известно из оригинальной работы Бенеша [3] (см. также [17]), условие (1.3) выполнено для любого $T>0$. Мы покажем, что этот же результат легко получить, не прибегая к технике кусочно-постоянной аппроксимации.

Утверждения теорем 2.1 и 2.2 принадлежат Бенешу. Их доказательства полностью новые.

Теорема 2.1. Пусть $M_{t}=M_{t}^{\prime}$ и выполнено условие (2.1). Тогда (1.3) выполнено для любого $T>0$.

Д о к аз а т е л ь с т в о. Выберем $\tau_{n}=\inf \left\{t:\left(\mathfrak{z}_{t} \vee B_{t}^{2}\right) \geqslant n\right\}^{1)}, n \geqslant 1$. Пишем

$$
\mathfrak{z}_{t \wedge \tau_{n}}=1+\int_{0}^{t} I_{\left\{s \leqslant \tau_{n}\right\}} \mathfrak{z}_{s} \sigma\left(B_{s}\right) d B_{s} .
$$

Определение $\tau_{n}$ и условие (2.1) гарантируют ограниченность подынтегральной функции в интеграле Ито $\int_{0}^{t} I_{\left\{s \leqslant \tau_{n}\right\} \mathfrak{z}_{s}} \sigma\left(B_{s}\right) d B_{s}$. Значит, $\mathfrak{z}_{t \wedge \tau_{n}}$ - квадратично интегрируемый мартингал и $\mathbf{E}_{\mathfrak{z}_{T \wedge \tau_{n}}}=1$. Если семейство $\left(\mathfrak{z}_{T \wedge \tau_{n}}\right)_{n \geqslant 1}$ равномерно интегрируемо, то $\mathbf{E}_{\mathfrak{z}_{T \wedge \tau_{n}}} \rightarrow 1, n \rightarrow \infty$, и $\lim _{n \rightarrow \infty} \mathbf{E}_{\mathfrak{z}_{T \wedge \tau_{n}}}=\mathbf{E}_{\mathfrak{z}_{T}}=1$. Таким образом, осталось проверить упомянутую равномерную интегрируемость. Следуя Хитсуде [11], воспользуемся теоремой Валле Пуссена с функцией $x \ln x, x \geqslant 0$, и покажем, что $\sup _{n} \mathbf{E}_{\mathfrak{z}_{T \wedge \tau_{n}}} \ln \left(\mathfrak{z}_{T \wedge \tau_{n}}\right)<\infty$. Это условие проверяется с помощью замены

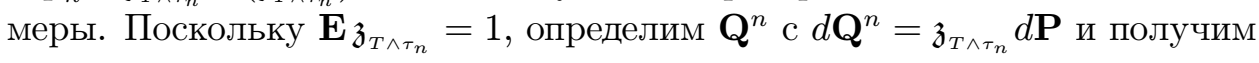

\footnotetext{
1) $\inf \{\varnothing\}=\infty$.
} 
(здесь $\widetilde{\mathbf{E}}^{n}$ математическое ожидание по мере $\left.\mathbf{Q}^{n}\right) \sup _{n} \mathbf{E}_{\mathfrak{z}_{T \wedge \tau_{n}}} \ln \left(\mathfrak{z}_{T \wedge \tau_{n}}\right)=$ $\sup _{n} \widetilde{\mathbf{E}}^{n} \ln \left(\mathfrak{z}_{T \wedge \tau_{n}}\right)$.

Далее,

$$
\ln \mathfrak{z}_{t}=\int_{0}^{t} \sigma\left(B_{s}\right) d B_{s}-\frac{1}{2} \int_{0}^{t} \sigma^{2}\left(B_{s}\right) d s \leqslant \int_{0}^{t} \sigma\left(B_{s}\right) d B_{s}
$$

влечет за собой неравенство

$$
\sup _{n} \mathbf{E}_{\mathfrak{z}_{T \wedge \tau_{n}}} \ln \left(\mathfrak{z}_{T \wedge \tau_{n}}\right) \leqslant \sup _{n} \widetilde{\mathbf{E}}^{n} \int_{0}^{T} I_{\left\{s \leqslant \tau_{n}\right\}} \sigma\left(B_{s}\right) d B_{s},
$$

которое подсказывает использование семимартингального представления $B_{t \wedge \tau_{n}}$ относительно $\mathbf{Q}^{n}$ в формуле $\widetilde{\mathbf{E}}^{n} \int_{0}^{T} I_{\left\{s \leqslant \tau_{n}\right\}} \sigma\left(B_{s}\right) d B_{s}$. Классическая теорема Гирсанова утверждает, что $B_{t \wedge \tau_{n}}=\int_{0}^{t} I_{\left\{s \leqslant \tau_{n}\right\}} \sigma\left(B_{s}\right) d s+\widetilde{B}_{t}^{n}$ с $\mathbf{Q}^{n}$-броуновским движением $\widetilde{B}_{t}^{n}$, остановленным в момент времени $\tau_{n}$, т.е. с квадратической вариацией $\langle\widetilde{B}\rangle_{t}=t \wedge \tau_{n}$. Отсюда следует неравенство

$$
\sup _{n} \mathbf{E}_{\mathfrak{z}_{T \wedge \tau_{n}}} \ln \left(\mathfrak{z}_{T \wedge \tau_{n}}\right) \leqslant \sup _{n} \widetilde{\mathbf{E}}^{n} \int_{0}^{T} I_{\left\{s \leqslant \tau_{n}\right\}} \sigma^{2}\left(B_{s}\right) d s,
$$

т.е. утверждение теоремы будет доказано, если мы проверим, что $\widetilde{\mathbf{E}}^{n} \int_{0}^{T} \sigma^{2}\left(B_{s \wedge \tau_{n}}\right) d s \leqslant \mathrm{r}$ с константой $\mathrm{r}$, не зависящей от $n$. Условие Бенеша (2.1) является ключевым в этой проверке. Оно позволяет заменить $\widetilde{\mathbf{E}}^{n} \int_{0}^{T} \sigma^{2}\left(B_{s \wedge \tau_{n}}\right) d s$ на $\widetilde{\mathbf{E}}^{n} \int_{0}^{T} B_{s \wedge \tau_{n}}^{2} d s$ и проверить только

$$
\widetilde{\mathbf{E}}^{n} \int_{0}^{T} B_{s \wedge \tau_{n}}^{2} d s \leqslant \mathrm{r}
$$

с константой $\mathrm{r}$, не зависящей от $n$. С этой целью применим формулу Ито к $B_{t \wedge \tau_{n}}^{2}$ и получим

$$
\begin{gathered}
B_{t \wedge \tau_{n}}^{2}=2 \int_{0}^{t} I_{\left\{s \leqslant \tau_{n}\right\}} B_{s} \sigma\left(B_{s}\right) d s+2 \int_{0}^{t} I_{\left\{s \leqslant \tau_{n}\right\}} B_{s} d \widetilde{B}_{s}^{n}+\left\langle\widetilde{B}^{n}\right\rangle_{t}, \\
\widetilde{\mathbf{E}}^{n} B_{t \wedge \tau_{n}}^{2}=2 \int_{0}^{t} \widetilde{\mathbf{E}}^{n} I_{\left\{s \leqslant \tau_{n}\right\}} B_{s} \sigma\left(B_{s}\right) d s+\widetilde{\mathbf{E}}^{n}\left\langle\widetilde{B}^{n}\right\rangle_{t} .
\end{gathered}
$$

Поскольку $\left|B_{s} \sigma\left(B_{s}\right)\right| \leqslant c\left[1+B_{s}^{2} \mid\right.$, имеет место оценка для $V_{t}^{n}=\widetilde{\mathbf{E}}^{n} B_{t \wedge \tau_{n}}^{2}$ :

$$
\widetilde{\mathbf{E}}^{n} B_{t \wedge \tau_{n}}^{2} \leqslant 2 \int_{0}^{t} \widetilde{\mathbf{E}}^{n} I_{\left\{s \leqslant \tau_{n}\right\}}\left|B_{s} \sigma\left(B_{s}\right)\right| d s+\widetilde{\mathbf{E}}^{n}\left(t \wedge \tau_{n}\right) .
$$

Поэтому $V_{t}^{n}$ удовлетворяет неравенству Гронуолла-Беллмана:

$$
V_{t}^{n} \leqslant \mathrm{r}\left[1+\int_{0}^{t} V_{s}^{n} d s\right]
$$

с подходящей положительной константой $r$.

Отсюда $\int_{0}^{t} V_{s}^{n} d s \leqslant e^{\mathrm{rt}}-1$, т.е. $\sup _{n} \widetilde{\mathbf{E}}^{n} \int_{0}^{T} B_{s \wedge \tau_{n}}^{2} d s \leqslant e^{\mathrm{r} T}-1$ Для любого $T>0$. Теорема доказана. 
Теорема 2.2. Пусть $M_{t}=M_{t}^{\prime \prime}$ и выполнено условие (2.2). Тогда $\mathbf{E}_{\mathfrak{z}_{T}}=1$ для любого $T>0$.

Д о к а з а т е л ь с т в о. Выберем $\tau_{n}=\inf \left\{t:\left(\mathfrak{z}_{t} \vee \sup _{s \leqslant t} B_{s}^{2}\right) \geqslant n\right\}$ и получим $\mathbf{E}_{\mathfrak{z}_{T \wedge \tau_{n}}}=1$. Теперь доказательство сводится к проверке неравенства $\sup _{n} \widetilde{\mathbf{E}}^{n} \ln \left(\mathfrak{z}_{T \wedge \tau_{n}}\right)<\infty$, где $\widetilde{\mathbf{E}}^{n}-$ математическое ожидание по мере $\mathbf{Q}^{n}, \mathbf{Q}^{n} \ll \mathbf{P}, d \mathbf{Q}^{n} / d \mathbf{P}=\mathfrak{z}_{T \wedge \tau_{n}}$. По теореме Гирсанова процесс $B_{t \wedge \tau_{n}}$ допускает представление относительно меры $\mathbf{Q}^{n}$ :

$$
B_{t \wedge \tau_{n}}=\int_{0}^{t} I_{\left\{s \leqslant \tau_{n}\right\}} \sigma_{s}(B) d s+\widetilde{B}_{t}^{n}
$$

с броуновским движением $\widetilde{B}_{t}^{n}$, остановленным в точке $\tau_{n}$, т.е. его квадратическая вариация $\langle\widetilde{B}\rangle_{t}=t \wedge \tau_{n}$.

Следовательно,

$$
\widetilde{\mathbf{E}}^{n} \int_{0}^{t} I_{\left\{s \leqslant \tau_{n}\right\}} \sigma_{s}(B) d B_{s}=\widetilde{\mathbf{E}}^{n} \int_{0}^{t} I_{\left\{s \leqslant \tau_{n}\right\}} \sigma_{s}^{2}(B) d s
$$

и, значит, в силу условия (2.2),

$$
\widetilde{\mathbf{E}}^{n} \int_{0}^{t} I_{\left\{s \leqslant \tau_{n}\right\}} \sigma_{s}^{2}(B) d s \leqslant \mathrm{r}\left[1+\widetilde{\mathbf{E}}^{n} \int_{0}^{t} I_{\left\{s \leqslant \tau_{n}\right\}} \sup _{s^{\prime} \leqslant s} B_{s^{\prime}}^{2} d s\right] .
$$

Итак, достаточно показать, что

$$
\sup _{n} \widetilde{\mathbf{E}}^{n} \int_{0}^{T} \sup _{s^{\prime} \leqslant s} B_{s^{\prime} \wedge \tau_{n}}^{2} d s<\infty .
$$

Используя представление (2.3) и неравенство Коши-Шварца-Буняковского, получаем

$$
\begin{aligned}
\sup _{t^{\prime} \leqslant t} B_{t^{\prime} \wedge \tau_{n}}^{2} & \leqslant 2\left|\int_{0}^{t} I_{\left\{s \leqslant \tau_{n}\right\}}\right| \sigma_{s}(B)|d s|^{2}+2 \sup _{t^{\prime} \leqslant t}\left|\widetilde{B}_{t^{\prime} \wedge \tau_{n}}^{n}\right|^{2} \\
& \leqslant 2 t \int_{0}^{t} I_{\left\{s \leqslant \tau_{n}\right\}} \sigma_{s}^{2}(B) d s+2 \sup _{t^{\prime} \leqslant t}\left|\widetilde{B}_{t^{\prime} \wedge \tau_{n}}^{n}\right|^{2} \\
& \leqslant 2 t \int_{0}^{t} I_{\left\{s \leqslant \tau_{n}\right\}} \mathrm{r}\left[1+\sup _{s^{\prime} \leqslant s} \sigma_{s^{\prime}}^{2}(B)\right] d s+2 \sup _{t^{\prime} \leqslant t}\left|\widetilde{B}_{t^{\prime} \wedge \tau_{n}}^{n}\right|^{2} .
\end{aligned}
$$

Кроме того, неравенство Дуба для квадратично интегрируемых мартингалов приводит к неравенству $\widetilde{\mathbf{E}}^{n} \sup _{t^{\prime} \leqslant t}\left|\widetilde{B}_{t^{\prime} \wedge \tau_{n}}^{n}\right|^{2} \leqslant 4\left(t \wedge \tau_{n}\right)$. Эти неравенства позволяют воспользоваться неравенством Гронуолла-Беллмана $V_{t}^{n} \leqslant \mathrm{r}\left[1+\int_{0}^{t} V_{s}^{n} d s\right], t \in[0, T]$, где $V_{t}^{n}:=\widetilde{\mathbf{E}}^{n} \sup _{t^{\prime} \leqslant t} B_{t^{\prime} \wedge \tau_{n}}^{2}$, и получить (2.4). Теорема доказана. 
3. Общая модель. Предположим, что мартингал $M=M^{c}+M^{d}$ участвует в конструкции некоторого семимартингала $X$. Естественно, такая модель не является абсолютно общей. Тем не менее она включает в себя многие примеры, известные в приложениях. С предлагаемой техникой доказательств согласуются не только обсуждаемые ниже примеры, но и другие модели. Мы не приводим ее здесь потому, что статья и так переполнена техническими деталями. Некоторые из них приводятся в п. 8.

Зафиксируем следующие обозначения и предположения:

- $\left(\Omega, F,\left(\mathscr{F}_{t}\right)_{t \in[0, T]}, \mathbf{P}\right)$ - стохастический базис со стандартными условиями;

- $\mathbf{C}=\mathbf{C}_{[0, T]}$ и $\mathbb{D}=\mathbb{D}_{[0, T]}$ - пространства непрерывных функций и пространства Скорохода функций, непрерывных справа и имеющих пределы слева;

- $x_{[0, t)}=\left\{x_{t^{\prime}}: t^{\prime}<t\right\}$ и $x_{[0, t]}=\left\{x_{t^{\prime}}: t^{\prime} \leqslant t\right\}$;

- $\left(B_{t}\right)_{t \in[0, T]}$ - стандартное броуновское движение;

- $\mu(d t, d z)$ - целочисленная случайная мера на $[0, T] \times \mathbf{R}_{+}$(см., например, [13] или [25]);

- $\nu(d t, d z):=d t K(d z)$ - компенсатор $\mu(d t, d z)$; здесь $K(d z)$ есть $\sigma$-конечная мера на $\mathbf{R}$ такая, что

$$
\int_{\mathbf{R}} z^{2} K(d z)<\infty
$$

- $a_{s}(x), b_{s}(x), \sigma_{s}(x)$ и $h_{s}(x, z), \varphi_{s}(x, z)$ - функции, измеримые относительно подходящей $\sigma$-алгебры, аргументы которых суть $\left(s, x_{s-}\right)$ и $\left(s, x_{[0, s)}, z\right)$, где $s \in[0, T], x_{[0, s)} \in \mathbb{D}_{[0, T]}, z \in \mathbf{R}$, причем для любого $x \in \mathbb{D}_{[0, T]}$ функции $a_{s}(x), b_{s}(x), \sigma_{s}(x)$ квадратично интегрируемы по $d s$ на $[0, T]$ и для любого $x \in \mathbb{D}_{[0, T]}$ и любого $z \in \mathbf{R}$ функции $h_{s}(x, z)$ и $\varphi_{s}(x, z)$ квадратично интегрируемы по $d s K(d z)$ на $[0, T] \times \mathbf{R} ;{ }^{2)}$ более того, предполагается, что

$$
\varphi_{s}(x, z)>-1 \quad\left(\Rightarrow \Delta M_{s}>-1\right) .
$$

- $\mathrm{r}$ - произвольная положительная константа, которая может принимать различные значения в разных ситуациях;

- $\inf \{\varnothing\}=\infty$.

Семимартингал $X$ предполагается единственным слабым решением уравнения Ито:

$$
\begin{aligned}
X_{t}= & X_{0}+\int_{0}^{t} a_{s}(X) d s+\int_{0}^{t} b_{s}(X) d B_{s} \\
& +\int_{0}^{t} \int_{\mathbf{R}} h_{s}(X, z)[\mu(d s, d z)-d s K(d z)] .
\end{aligned}
$$

2) В случае взрыва эти свойства предполагаются выполненными до момента взрыва. 
Мартингал $M=M^{c}+M^{d}$ порождается этим уравнением, т.е.

$$
M_{t}=\int_{0}^{t} \sigma_{s}(X) d B_{s}+\int_{0}^{t} \int_{\mathbf{R}} \varphi_{s}(X, z)[\mu(d s, d z)-d s K(d z)]=: M_{t}^{c}+M_{t}^{d} .
$$

4. $X$ - не взрывающийся марковский процесс.

4.1. Формулировка основного результата. Примеры. Поскольку $X$ - марковский процесс, уточним его описание, т.е. заменим (3.1) таким уравнением:

$$
\begin{aligned}
X_{t}= & X_{0}+\int_{0}^{t} a_{s}\left(X_{s-}\right) d s+\int_{0}^{t} b_{s}\left(X_{s-}\right) d B_{s} \\
& +\int_{0}^{t} \int_{\mathbf{R}} h_{s}\left(X_{s-}, z\right)[\mu(d s, d z)-d s K(d z)],
\end{aligned}
$$

где $a_{s}\left(X_{s-}\right):=a\left(s, X_{s-}\right), b_{s}\left(X_{s-}\right):=b\left(s, X_{s-}\right)$ и $h_{s}\left(X_{s-}, z\right):=h\left(s, X_{s-}, z\right)$.

Аналогичным образом преобразуем (1.1):

$$
\begin{aligned}
\mathfrak{z}_{t} & =1+\int_{0}^{t} \mathfrak{z}_{s-} d M_{s} \\
& =1+\int_{0}^{t} \mathfrak{z}_{s-}\left[\sigma_{s}\left(X_{s-}\right) d B_{s}+\int_{\mathbf{R}} \varphi_{s}\left(X_{s-}, z\right)[\mu(d s, d z)-d s K(d z)]\right] .
\end{aligned}
$$

Мы будем иметь дело с двумя операторами, зависящими от $s$ : $L_{s}\left(x_{s-}\right)$ и $\mathfrak{L}_{s}\left(x_{s-}\right)$, преобразующими $\left(x_{s}\right)_{s \in[0, T]} \in \mathbb{D}$ следующим образом:

$$
\begin{aligned}
L_{s}\left(x_{s-}\right):= & 2 x_{s-} a_{s}\left(x_{s-}\right)+b_{s}^{2}\left(x_{s-}\right)+\int_{\mathbf{R}} h_{s}^{2}\left(x_{s-}, z\right) \\
\mathfrak{L}_{s}\left(x_{s-}\right):= & 2 x_{s-}\left[a_{s}\left(x_{s-}\right)+b_{s}\left(x_{s-}\right) \sigma_{s}\left(x_{s-}\right)+\int_{\mathbf{R}} h_{s}\left(x_{s-}, z\right) \varphi_{s}\left(x_{s-}, z\right) K(d z)\right] \\
& +b_{s}^{2}\left(x_{s-}\right)+\int_{\mathbf{R}} h_{s}^{2}\left(x_{s-}, z\right) K(d z) \\
& +\int_{\mathbf{R}} h_{s}^{2}\left(x_{s-}, z\right) \varphi_{s}\left(x_{s-}, z\right) K(d z) .
\end{aligned}
$$

Роль $L_{s}\left(x_{s-}\right)$ объясняется в следующем утверждении.

Предложение 4.1. Пусть $X_{0}^{2} \leqslant \mathrm{r} u L_{s}\left(x_{s-}\right) \leqslant \mathrm{r}\left[1+x_{s-}^{2}\right]$. Тогда

$$
\sup _{t \in[0, T]} \mathbf{E} X_{t}^{2}<\infty
$$

т.е. прочесс $X$ не взрывается на любом временном интервале $[0, T]$.

Д о к а з а т е л ь с т в о. Применяя формулу Ито к $X_{t}^{2}$, получаем

$$
X_{t}^{2}=X_{0}^{2}+\int_{0}^{t} L_{s}\left(X_{s-}\right) d s+\mathscr{M}_{t}
$$


где $\mathscr{M}_{t}$ - локальный мартингал. Заметим, что определения оператора $L_{s}\left(x_{s-}\right)$ и момента $\zeta_{n}:=\inf \left\{t: X_{t}^{2} \geqslant n\right\}$ обеспечивают $\mathbf{E} X_{t \wedge \zeta_{n}}^{2}<\infty$ и, поскольку

$$
X_{t \wedge \zeta_{n}}^{2}=X_{0}^{2}+\int_{0}^{t} I_{\left\{s \leqslant \zeta_{n}\right\}} L_{s}\left(X_{s-}\right) d s+\mathscr{M}_{t \wedge \zeta_{n}},
$$

то $\mathbf{E} \mathscr{M}_{t \wedge \tau_{n}}^{2}<\infty$ и $\mathbf{E} \mathscr{M}_{t \wedge \zeta_{n}}=0$. Воспользуемся теперь тем, что функция $V_{t}^{n}=\mathbf{E} X_{t \wedge \zeta_{n}}^{2}$ является решением неравенства Гронуолла-Беллмана: $V_{t}^{n} \leqslant \mathrm{r}\left[1+\int_{0}^{t} V_{s}^{n} d s\right]$. Отсюда $\mathbf{E} X_{t \wedge \zeta_{n}}^{2} \leqslant r e^{\mathrm{rt}}$ и по лемме Фату $\mathbf{E} X_{t}^{2} \leqslant \mathrm{r} e^{\mathrm{r} t}$.

Значит, $\sup _{t \in[0, T]} \mathbf{E} X_{t}^{2} \leqslant r e^{\mathrm{r} T}$. Предложение доказано.

3 а м е ч а н и е 2. Забегая вперед, можно пояснить роль оператора $\mathfrak{L}_{s}\left(x_{s-}\right)$. Предположим, что $\mathbf{E}_{\mathfrak{z}_{T}}=1$. Тогда мера $\mathbf{Q} \ll \mathbf{P}$ с $d \mathbf{Q} / \mathbf{d} \mathbf{P}=\mathfrak{z}_{\text {T }}$ является вероятностной мерой. В этом случае оператор $\mathfrak{L}_{s}\left(x_{s-}\right)$ играет роль оператора $L_{s}\left(x_{s-}\right)$ для процесса $X_{t}$ по мере $\mathbf{Q}$.

Теорема 4.1. Пусть $\left|X_{0}\right| \leqslant \mathrm{r} u$

1) $\sigma_{s}^{2}\left(x_{s-}\right)+\int_{\mathbf{R}} \varphi_{s}^{2}\left(x_{s-}, z\right) K(d z) \leqslant \mathrm{r}\left[1+x_{s-}^{2}\right]$,

2) $L_{s}\left(x_{s-}\right) \leqslant \mathrm{r}\left[1+x_{s-}^{2}\right]$,

3) $\mathfrak{L}_{s}\left(x_{s-}\right) \leqslant \mathrm{r}\left[1+x_{s-}^{2}\right]$.

Тогда $\left(\mathfrak{z}_{t}\right)_{t \in[0, T]}$ является мартингалом для любого $T>0$.

Доказательство теоремы приводится в п. 4.2. Ее применимость для разнообразных моделей иллюстрируется ниже.

Для упрощения текста мы будем заменять слова «условия теоремы

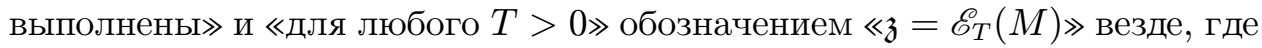
это не приводит к недоразумению.

П р и м е р 4.1 (см. [28]). $X$ - чисто разрывный мартингал с независимыми приращениями. Пусть

$$
\begin{aligned}
X_{t} & =\int_{0}^{t} \int_{\mathbf{R}} z[\mu(d s, d z)-K(d z) d s], \\
M_{t} & =\int_{0}^{t} \int_{\mathbf{R}}\left|X_{s-} z\right|[\mu(d s, d z)-K(d z) d s] .
\end{aligned}
$$

Поскольку а) $X_{0}=0$,

b) $a_{s}\left(x_{s-}\right)=b_{s}\left(x_{s-}\right)=0$ и $h_{s}\left(x_{s-}, z\right)=z$,

c) $\sigma_{s}\left(x_{s-}\right)=0$ и $\varphi_{s}\left(x_{s-}, z\right)=\left|x_{s-} z\right|$,

d) $\int_{\mathbf{R}} z^{2} K(d z)<\infty$

то, учитывая дополнительное условие $\int_{\mathbf{R}}|z|^{3} K(d z)<\infty$, получаем, что каждая из функций

$$
\begin{gathered}
\sigma_{s}^{2}\left(x_{s-}\right)+\int_{\mathbf{R}} \varphi_{s}^{2}\left(x_{s-}, z\right) K(d z)=x_{s-}^{2} \int_{\mathbf{R}} z^{2} K(d z), \quad L_{s}\left(x_{s-}\right)=\int_{\mathbf{R}} z^{2} K(d z), \\
\mathfrak{L}_{s}\left(x_{s-}\right)=2 x_{s-}\left|x_{s-}\right| \int_{\mathbf{R}} z|z| K(d z)+\int_{\mathbf{R}} z^{2} K(d z)+\left|x_{s-}\right| \int_{\mathbf{R}}|z|^{3} K(d z)
\end{gathered}
$$

не превосходит $\mathrm{r}\left[1+x_{s-}^{2}\right]$. 
Итак, $\mathfrak{z}=\mathscr{E}_{T}(M)$.

$\Pi$ р и м е р 4.2. Модель CEV (Constant Elasticity of Variance $[1],[6],[8])$. Пусть

$$
X_{t}=1+\int_{0}^{t} X_{s} d s+\int_{0}^{t} \sqrt{X_{s}^{+}} d B_{s} \quad \text { и } \quad M_{t}=\int_{0}^{t} \sqrt{X_{s}^{+}} d B_{s} .
$$

Тогда

a) $X_{0}=1$

b) $a_{s}\left(x_{s-}\right)=x_{s-}, b_{s}\left(x_{s-}\right)=\sqrt{x_{s-}^{+}}$и $h_{s}\left(x_{s-}, z\right)=0$,

c) $\sigma_{s}\left(x_{s-}\right)=\sqrt{x_{s-}^{+}}$и $\varphi_{s}\left(x_{s-}, z\right)=0$.

Следовательно, каждая из функций

$$
\begin{gathered}
\sigma_{s}^{2}\left(x_{s-}\right)+\int_{\mathbf{R}} \varphi_{s}^{2}\left(x_{s-}, z\right) K(d z)=x_{s-}^{+}, \\
L_{s}\left(x_{s-}\right)=2 x_{s-}^{2}+x_{s-}^{+}, \quad \mathfrak{L}_{s}\left(x_{s-}\right)=2 x_{s-}\left[x_{s-}+x_{s-}^{+}\right]+x_{s-}^{+}
\end{gathered}
$$

не превосходит $\mathrm{r}\left[1+x_{s-}^{2}\right]$. Следует отметить, что $X_{t}=X_{t \wedge \vartheta}$, где $\vartheta=$ $\inf \left\{t: X_{t}=0\right\}-$ момент поглощения в нуле. Поэтому $\left(\mathfrak{z}_{t \wedge \vartheta}\right)_{t \in[0, T]}-$ мартингал для любого $T>0$.

П р и м е р 4.3. Устойчивый снос. Пусть

$$
X_{t}=1-\int_{0}^{t} X_{s}^{3} d s+\int_{0}^{t} X_{s} d B_{s} \quad \text { и } \quad M_{t}=\int_{0}^{t} X_{s} d B_{s} .
$$

Тогда

a) $X_{0}=1$

b) $a_{s}\left(x_{s-}\right)=-x_{s-}^{3}, b_{s}\left(x_{s-}\right)=x_{s-}$ и $h_{s}\left(x_{s-}, z\right)=0$,

c) $\sigma_{s}\left(x_{s-}\right)=x_{s-}^{+}$и $\varphi_{s}\left(x_{s-}, z\right)=0$

и, значит, каждая из функций

$$
\sigma_{s}^{2}\left(x_{s-}\right)=\left(x_{s}^{+}\right)^{2}, \quad L_{s}\left(x_{s-}\right)=-2 x_{s}^{4}+x_{s}^{2}, \quad \mathfrak{L}_{s}\left(x_{s-}\right)=-2 x_{s}^{4}+x_{s}^{2}+2\left|x_{s}^{3}\right|
$$

не превосходит $\mathrm{r}\left[1+x_{s-}^{2}\right]$. Итак, $\mathfrak{z}=\mathscr{E}_{T}(M)$.

П р и м е р 4.4. Броуновский мост. Гауссовский процесс $X_{t}$ с нулевым средним, заданный на интервале $[0,1]$, называется броуновским мостом, если его корреляционная функция имеет вид

$$
R\left(t^{\prime}, t\right)=\left(t^{\prime} \wedge t\right)\left[1-\left(t^{\prime} \vee t\right)\right]
$$

Известно, что $X_{t}$ является единственным решением уравнения Ито

$$
X_{t}=-\int_{0}^{t} \frac{X_{s}}{1-s} d s+B_{t}, \quad t \in[0,1), \quad \lim _{t \uparrow 1} X_{t}=0 .
$$

Пусть $M_{t}=\int_{0}^{t} X_{s} d B_{s}, t \leqslant 1$. 
Здесь $T=1$ и
a) $X_{0}=0$,
b) $a_{s}\left(x_{s-}\right)=-x_{s-} /(1-s)(s<1), b_{s}\left(x_{s-}\right)=x_{s-}$ и $h_{s}\left(x_{s-}, z\right)=0$,
c) $\sigma_{s}\left(x_{s-}\right)=x_{s-}$ и $\varphi_{s}\left(x_{s-}, z\right)=0$.

Поэтому каждая из функций

$\sigma_{s}^{2}\left(x_{s-}\right)=x_{s-}^{2}, \quad L_{s}\left(x_{s-}\right)=-2 x_{s-}^{4}+x_{s-}^{2}, \quad \mathfrak{L}_{s}\left(x_{s-}\right)=-2 x_{s-}^{4}+x_{s}^{2}+2\left|x_{s-}^{3}\right|$

не превосходит $\mathrm{r}\left[1+x_{s-}^{2}\right]$ и, по теореме $4.1, \mathbf{E}_{\mathfrak{z}_{1}}=1$.

П р и м е р 4.5. Одно обобщение из [29]. Пусть $\alpha \in(-1,0]$ и

$$
X_{t}=1+\int_{0}^{t}\left|X_{s}\right|^{\alpha} d s+B_{t} \quad \text { и } \quad M_{t}=\int_{0}^{t} X_{s} d B_{s} .
$$

В [29] показано, что $\mathfrak{z}=\mathscr{E}_{T}(M)$.

Теорема 4.1 позволяет доказать, что $\mathfrak{z}=\mathscr{E}_{T}(M)$, даже если $\alpha=-1$. Действительно, при $\alpha=1$ процесс $X_{t}$ является процессом Бесселя (см., например, [32, упражнение 2.25 , с. 197]), т.е.

$$
X_{t}=1+\int_{0}^{t} \frac{d s}{X_{s}}+B_{t}
$$

Тогда

a) $X_{0}=1$,

b) $a_{s}\left(x_{s_{-}}\right)=1 /\left(x_{s-} \vee 0\right), b_{s}\left(x_{s-}\right)=1$ и $h_{s}\left(x_{s-}, z\right)=0$,

c) $\sigma_{s}\left(x_{s-}\right)=x_{s-}$ и $\varphi_{s}\left(x_{s-}, z\right)=0$

и каждая из функций

$$
\sigma_{s}^{2}\left(x_{s-}\right)=x_{s-}^{2}, \quad L_{s}\left(x_{s-}\right)=3, \quad \mathfrak{L}_{s}\left(x_{s-}\right)=3+2 x_{s-}
$$

не превосходит $\mathrm{r}\left[1+x_{s-}^{2}\right]$. Поэтому $\mathfrak{z}=\mathscr{E}_{T}(M)$.

5. Семимартингал $X$, не являющийся марковским процесcom.

5.1. Формулировка основного результата. Примеры. Несмотря на то что мы остаемся в рамках $(3.1)$ и $(3.2)$, операторы $L_{s}(x)$ и $\mathfrak{L}_{s}(x)$ изменяются следующим образом:

$$
L_{s}(x):=a_{s}^{2}(x)+b_{s}^{2}(x)+\int_{\mathbf{R}} h_{s}^{2}(x, z) K(d z),
$$

и

$$
\begin{aligned}
\mathfrak{L}_{s}(x):= & a_{s}^{2}(x)+b_{s}^{2}(x)+\int_{\mathbf{R}} h_{s}^{2}(x, z) K(d z)+b_{s}^{2}(x) \sigma_{s}^{2}(x) \\
& +\int_{\mathbf{R}} h_{s}^{2}(x, z) K(d z) \int_{\mathbf{R}} \varphi_{s}^{2}(x, z) K(d z) \\
& +\int_{\mathbf{R}} h_{s}^{2}(x, z) \varphi_{s}(x, z) K(d z) .
\end{aligned}
$$


Теорема 5.1. Если $\left|X_{0}\right| \leqslant \mathrm{r} u$

1) $\sigma_{s}^{2}(x)+\int_{\mathbf{R}} \varphi_{s}^{2}(x, z) K(d z) \leqslant r\left[1+\sup _{s^{\prime}<s} x_{s^{\prime}}^{2}\right]$

2) $L_{s}(x) \leqslant r\left[1+\sup _{s^{\prime}<s} x_{s^{\prime}}^{2}\right]$,

3) $\mathfrak{L}_{s}(x) \leqslant r\left[1+\sup _{s^{\prime}<s} x_{s^{\prime}}^{2}\right]$, $m o \mathfrak{z}=\mathscr{E}_{T}(M)$.

Доказательство теоремы приводится в п.6.3. Заметим, что процесс $X$ не взрывается в силу условия 2). Ниже мы рассмотрим примеры, обслуживаемые этой теоремой.

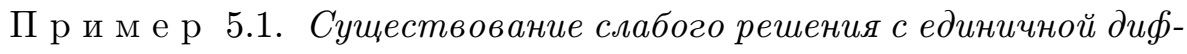
фузией. Стохастическое дифференциальное уравнение

$$
X_{t}=\int_{0}^{t} a_{s}(X) d s+B_{t}
$$

допускает слабое решение на любом конечном интервале $[0, T]$, если на этом интервале

$$
a_{s}^{2}(y) \leqslant r\left[1+\sup _{s^{\prime} \leqslant s} y_{s}^{2}\right], \quad\left(y_{s}\right)_{s \in[0, T]} \in \mathbf{C}
$$

(cp. с условиями теоремы 7.2 в [26, гл. $7, \S 7.2]$ ).

Положим $M_{t}=\int_{0}^{t} \sigma_{s}(B) d B_{t}$, где $\sigma_{s}(y) \equiv a_{s}(y)$. Тогда по теореме $5.1 \mathbf{E}_{\mathfrak{z}_{T}}=1$ и, значит, существует вероятностная мера $\mathbf{Q} \ll \mathbf{P}$ с $d \mathbf{Q} / d \mathbf{P}=\mathfrak{z}_{T}$. Поэтому, по теореме Гирсанова, процесс $\left(B_{t}, \mathbf{Q}\right)_{t \in[0, T]}$ является слабым решением уравнения Ито $B_{t}=\int_{0}^{t} a_{s}(B) d s+\widetilde{B}_{t}$ c Qброуновским движением $\widetilde{B}_{t}$.

Отметим также слабую единственность этого решения (см. [26, гл. 4, теорема 4.12]).

П р и м е р 5.2. Существование слабого решения с вырожденной диффузией. Предположим, что уравнение Ито

$$
Y_{t}=X_{0}+\int_{0}^{t} b_{s}(Y) d B_{s}
$$

с $b_{s}^{2}(y) \geqslant 0$ имеет слабое решение.

Теорема 5.1 позволяет заменить вероятностную меру таким образом, чтобы по новой мере уравнение Ито

$$
X_{t}=X_{0}+\int_{0}^{t} a_{s}(X) d s+\int_{0}^{t} b_{s}(X) d B_{s}
$$

также допускало слабое решение.

Это утверждение имеет место, если
a) $X_{0}^{2} \leqslant r$
b) $a_{s}(x)=a_{s}(x) I_{\left\{b^{2}(x)>0\right\}}$, 
c) каждая из функций $a_{s}^{2}(x), b_{s}^{2}(x),\left(a_{s}^{2}(x) / b_{s}^{2}(x)\right) I_{\left\{b_{s}^{2}(x)>0\right\}}$ не превосходит $r\left[1+\sup _{s^{\prime} \leqslant s} x_{s^{\prime}}^{2}\right], s \in[0, T], \quad\left(x_{s}\right)_{s \in[0, T]} \in \mathbf{C}$ (cр. с теоремой 7.19 в $[26$, гл. $7, \S 7.6])$. Для этого достаточно выбрать $\sigma_{s}(x)=$ $\left(a_{s}(x) / b_{s}(x)\right) I_{\left\{b_{s}^{2}(x)>0\right\}}$ и $M_{t}=\int_{0}^{t} \sigma_{s}(B) d B_{s}$ и применить теорему 5.1 для $\mathfrak{z}_{t}=1+\int_{0}^{t} \mathfrak{z}_{s} \sigma_{s}(x) d B_{s}$. Поскольку $\mathbf{E}_{\mathfrak{z}_{T}}=1$, существует мера $\mathbf{Q} \ll \mathbf{P}$ с плотностью $d \mathbf{Q} / d \mathbf{P}=\mathfrak{z}_{T}$. Тогда в силу теоремы Гирсанова

$$
X_{t}=X_{0}+\int_{0}^{t} \underbrace{b_{s}(X) \sigma_{s}(X)}_{=a_{s}(X)} d s+\int_{0}^{t} b_{s}(X) d \widetilde{B}_{s}
$$

с Q-броуновским движением $\widetilde{B}_{t}$.

П р и м е р 5.3. Уравнение Ито с запаздьванием. Теорема 5.1 применима к стохастической модели с запаздыванием, часто используемой в современной теории управления.

Пусть $\vartheta>0$ обозначает фиксированный параметр запаздывания в уравнении

$$
X_{t}=I_{\left\{X_{u} \in[-\vartheta, 0]\right\}}+\int_{0}^{t} a_{s}\left(X_{s-\vartheta}\right) d s+\int_{0}^{t} b_{s}\left(X_{s-\vartheta}\right) d B_{s} .
$$

Пусть $M_{t}=\int_{0}^{t} \sigma_{s}\left(X_{s-\vartheta}\right) d B_{s}$ и, значит, $\mathfrak{z}_{t}=1+\int_{0}^{t} \mathfrak{z}_{s} \sigma_{s}\left(X_{s-\vartheta}\right) d B_{s}$.

Тогда по теореме $5.1 \mathfrak{z}=\mathscr{E}_{T}(M)$ при условии, что каждая из функций $a_{s}^{2}\left(x_{s-\vartheta}\right)+b_{s}^{2}\left(x_{s-\vartheta}\right), \sigma_{s}^{2}\left(x_{s-\vartheta}\right), \sigma_{s}^{2}\left(x_{s-\vartheta}\right) b_{s}^{2}\left(x_{s-\vartheta}\right)$ не превосходит $\mathrm{r}\left[1+\sup _{s^{\prime} \leqslant s} x_{s^{\prime}}^{2}\right], s \in[0, T],\left(x_{s}\right)_{s \in[0, T]} \in \mathbf{C}$.

\section{6. Доказательства.}

6.1. Вспомогательный результат.

Теоремы 4.1 и 5.1 используют одну и ту же идею доказательства, которая состоит в проверке равномерной интегрируемости семейства $\left\{\mathfrak{z}_{T \wedge \tau_{n}}\right\}_{n \rightarrow \infty}$, выраженной в форме

$$
\sup _{n} \mathbf{E}_{\mathfrak{z}_{T \wedge \tau_{n}}} \ln \left(\mathfrak{z}_{T \wedge \tau_{n}}\right)<\infty \text {. }
$$

Так как $X, M$ определены в (3.1), (3.2) соответственно, то процесс (1.2), где мартингалы $M_{t}^{c}$ и $M_{t}^{d}$ определены в (3.2), является единственным решением уравнения

$$
\mathfrak{z}_{t}=1+\int_{0}^{t} \mathfrak{z}_{s-} \sigma_{s}(X) d B_{s}+\int_{0}^{t} \int_{\mathbf{R}} \mathfrak{z}_{s-} \varphi_{s}(X, z)[\mu(d s, d z)-d s K(d z)] .
$$

Последовательности моментов остановки $\tau_{n}$ определяются следующим образом:

$$
\begin{array}{ll}
\tau_{n}=\inf \left\{t:\left(\mathfrak{z}_{t} \vee X_{t}^{2}\right) \geqslant n\right\} & \text { в теореме 4.1, } \\
\tau_{n}=\inf \left\{t:\left(\mathfrak{z}_{t} \vee \sup _{s \leqslant t} X_{s}^{2}\right) \geqslant n\right\} & \text { в теореме 5.1. }
\end{array}
$$


Заметим также, что $\mathfrak{z}\left(s \wedge \tau_{n}\right)$ - и $X_{\left(s \wedge \tau_{n}\right) \text { - }}^{2}$ являются ограниченными процессами. Следовательно,

$$
\begin{aligned}
\mathfrak{z}_{t \wedge \tau_{n}}= & 1+\int_{0}^{t} I_{\left\{s \leqslant \tau_{n}\right\} \mathfrak{z}_{\left(s \wedge \tau_{n}\right)-}} \sigma_{s}(X) d B_{s} \\
& +\int_{0}^{t} \int_{\mathbf{R}} I_{\left\{s \leqslant \tau_{n}\right\} \mathfrak{z}_{\left(s \wedge \tau_{n}\right)-}} \varphi_{s}(X, z)[\mu(d s, d z)-d s K(d z)],
\end{aligned}
$$

что влечет за собой

$$
\mathbf{E}\left(\mathfrak{z}_{T \wedge \tau_{n}}-1\right)^{2}=\mathbf{E} \int_{0}^{T} I_{\left\{s \leqslant \tau_{n}\right\}} \mathfrak{z}_{\left(s \wedge \tau_{n}\right)-}^{2}\left(\sigma_{s}^{2}(X)+\int_{\mathbf{R}} \varphi_{s}^{2}(X, z) K(d z)\right) d s .
$$

Поэтому случайный процесс $\left(\mathfrak{z}_{t \wedge \tau_{n}}\right)_{t \in[0, T]}$ является квадратично интегрируемым мартингалом с $\mathbf{E}_{\mathfrak{z}_{t \wedge \tau_{n}}}=1$.

По аналогии с (2.1) и (2.2) предположим, что для любого $s \in[0, T]$, $\left(x_{s}\right)_{s \leqslant T} \in \mathbb{D}$

$$
\sigma_{s}^{2}(x)+\int_{\mathbf{R}} \varphi_{s}^{2}(x, z) K(d z) \leqslant \begin{cases}\mathrm{r}\left[1+x_{s-}^{2}\right] & \text { в теореме 4.1 } \\ \mathrm{r}\left[1+\sup _{s^{\prime}<s} x_{s^{\prime}}^{2}\right] & \text { в теореме 5.1. }\end{cases}
$$

Лемма 6.1. Семейство $\left\{\mathfrak{z}_{T \wedge \tau_{n}}\right\}_{n \geqslant 1}$ равномерно интегрируемо, если

$$
\left.\begin{array}{ll}
\sup _{n} \widetilde{\mathbf{E}}^{n} \int_{0}^{T} X_{s \wedge \tau_{n}}^{2} d s & \text { в теореме } 4.1 \\
\sup _{n} \widetilde{\mathbf{E}}^{n} \int_{0}^{T} \sup _{s^{\prime}<s} X_{s^{\prime} \wedge \tau_{n}}^{2} d s & \text { в теореме } 5.1
\end{array}\right\}<\infty .
$$

Д о к а з а т е л ь с т в о. Существование вероятностной меры $\mathbf{Q}^{n} \ll$

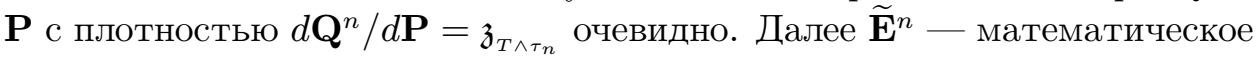
ожидание, соответствующее мере $\mathbf{Q}^{n}$. Равномерная интегрируемость семейства случайных величин $\left\{\mathfrak{z}_{T \wedge \tau_{n}}\right\}_{n \rightarrow \infty}$ проверятся с помощью теоремы Валле-Пуссена, в которой используется функция $x \ln x, x \geqslant 0$, такая же, как в доказательстве теорем 2.1 и 2.2 , т.е. проверяется

$$
\sup _{n} \mathbf{E}_{\mathfrak{z}_{T \wedge \tau_{n}}} \ln \left(\mathfrak{z}_{T \wedge \tau_{n}}\right)<\infty .
$$

Эта формула удобна, поскольку имеет место представление

$$
\mathfrak{z}_{t \wedge \tau_{n}}=\exp \left(M_{t \wedge \tau_{n}}-A_{t \wedge \tau_{n}}\right),
$$

в котором $M_{t \wedge \tau_{n}}$ - квадратично интегрируемый мартингал и $A_{t \wedge \tau_{n}}-$ возрастающий положительный процесс:

$$
\begin{aligned}
M_{t \wedge \tau_{n}}= & \int_{0}^{t} I_{\left\{s \leqslant \tau_{n}\right\}} \sigma_{s}(X) d B_{s}+\int_{0}^{t} \int_{\mathbf{R}} I_{\left\{s \leqslant \tau_{n}\right\}} \varphi_{s}(X, z)[\mu(d s, d z)-d s K(d z)], \\
A_{t \wedge \tau_{n}}= & \frac{1}{2} \int_{0}^{t} I_{\left\{s \leqslant \tau_{n}\right\}} \sigma_{s}^{2}(X) d s \\
& +\int_{0}^{t} \int_{\mathbf{R}} I_{\left\{s \leqslant \tau_{n}\right\}}\left\{\varphi_{s}(X, z)-\ln \left[1+\varphi_{s}(X, z)\right]\right\} \mu(d s, d z) .
\end{aligned}
$$


Условие $\varphi_{s}(X, z)>-1$ гарантирует неравенство

$$
\varphi_{s}(X, z)-\ln \left[1+\varphi_{s}(X, z)\right] \geqslant 0,
$$

которое вместе с $\sigma_{s}^{2}(X) \geqslant 0$ обеспечивает упомянутое свойство $A_{t \wedge \tau_{n}} \geqslant 0$. Поэтому $\ln \left(\mathfrak{z}_{T \wedge \tau_{n}}\right) \leqslant M_{T \wedge \tau_{n}}$ и, следовательно,

$$
\mathfrak{z}_{T \wedge \tau_{n}} \ln \left(\mathfrak{z}_{T \wedge \tau_{n}}\right) \leqslant \mathfrak{z}_{T \wedge \tau_{n}} M_{T \wedge \tau_{n}} .
$$

Оба процесса $\mathfrak{z}_{t \wedge \tau_{n}}$ и $M_{t \wedge \tau_{n}}$ являются квадратично интегрируемыми мартингалами, допускающими разложения $\mathfrak{z}_{t \wedge \tau_{n}}=\mathfrak{z}_{t \wedge \tau_{n}}^{c}+\mathfrak{z}_{t \wedge \tau_{n}}^{d}$ и $M_{t \wedge \tau_{n}}=$ $M_{t \wedge \tau_{n}}^{c}+M_{t \wedge \tau_{n}}^{d}$, в которых

$$
\begin{gathered}
\mathfrak{z}_{T \wedge \tau_{n}}^{c}=\int_{0}^{T} I_{\left\{s \leqslant \tau_{n}\right\} \mathfrak{z}_{s-}} \sigma_{s}(X) d B_{s}, \quad M_{T \wedge \tau_{n}}^{c}=\int_{0}^{T} I_{\left\{s \leqslant \tau_{n}\right\}} \sigma_{s}(X) d B_{s}, \\
\mathfrak{z}_{T \wedge \tau_{n}}^{d}=1+\int_{0}^{T} \int_{\mathbf{R}} I_{\left\{s \leqslant \tau_{n}\right\}} \mathfrak{z}_{s-} \varphi_{s}(X, z)[\mu(d s, d z)-d s K(d z)], \\
M_{T \wedge \tau_{n}}^{d}=\int_{0}^{T} \int_{\mathbf{R}} I_{\left\{s \leqslant \tau_{n}\right\}} \varphi_{s}(X, z)[\mu(d s, d s)-d s K(d z)] .
\end{gathered}
$$

Поэтому $\mathbf{E} M_{T \wedge \tau_{n}} \mathfrak{z}_{T \wedge \tau_{n}}=\widetilde{\mathbf{E}}^{n} M_{T \wedge \tau_{n}}$. Очевидно, что

$$
\begin{aligned}
\mathbf{E} M_{T \wedge \tau_{n}}^{c, n} \mathfrak{z}_{T \wedge \tau_{n}} & =\mathbf{E} \int_{0}^{T} I_{\left\{s \leqslant \tau_{n}\right\} \mathfrak{z}_{s}} \sigma_{s}^{2}(X) d s=\mathbf{E}_{\mathfrak{z}_{T \wedge \tau_{n}}} \int_{0}^{T} I_{\left\{s \leqslant \tau_{n}\right\}} \sigma_{s}^{2}(X) d s \\
& =\widetilde{\mathbf{E}}^{n} \int_{0}^{T} I_{\left\{s \leqslant \tau_{n}\right\}} \sigma_{s}^{2}(X) d s, \\
\mathbf{E} M_{T \wedge \tau_{n}}^{d, n} \mathfrak{z}_{T \wedge \tau_{n}} & =\mathbf{E} \int_{0}^{T} \int_{\mathbf{R}} I_{\left\{s \leqslant \tau_{n}\right\}} \mathfrak{z}_{s-} \varphi_{s}^{2}(X, z) K(d z) d s \\
& =\widetilde{\mathbf{E}}^{n} \int_{0}^{T} \int_{\mathbf{R}} I_{\left\{s \leqslant \tau_{n}\right\}} \varphi_{s}^{2}(X) K(d z) d s .
\end{aligned}
$$

Следовательно,

$$
\widetilde{\mathbf{E}}^{n} M_{T \wedge \tau_{n}}=\widetilde{\mathbf{E}}^{n} \int_{0}^{T} I_{\left\{s \leqslant \tau_{n}\right\}}\left(\sigma_{s}^{2}(X)+\int_{\mathbf{R}} \varphi_{s}^{2}(X, z) K(d z)\right) d s .
$$

Теперь, принимая во внимание (6.3), завершаем доказательство:

$$
\sup _{n} \mathbf{E}_{\mathfrak{z}_{T \wedge \tau_{n}}} \ln \left(\mathfrak{z}_{T \wedge \tau_{n}}\right) \leqslant \mathrm{r} \begin{cases}T+\sup _{n} \widetilde{\mathbf{E}}^{n} \int_{0}^{T} X_{s \wedge \tau_{n}}^{2} d s, & \text { в теореме 4.1, } \\ T+\sup _{n} \widetilde{\mathbf{E}}^{n} \int_{0}^{T} \sup _{s^{\prime} \leqslant s} X_{s^{\prime} \wedge \tau_{n}}^{2} d s & \text { в теореме 5.1 }\end{cases}
$$

при условии, что выполнены условия леммы. Лемма доказана. 
6.2. Доказательство теоремы 4.1. В силу леммы 6.1 утверждение теоремы имеет место, если выполнено условие

$$
\sup _{n} \widetilde{\mathbf{E}}^{n} \int_{0}^{T} X_{s \wedge \tau_{n}}^{2} d s<\infty
$$

с $\tau_{n}$ из (6.1), согласованным с теоремой 4.1. Поскольку математическое ожидание $\widetilde{\mathbf{E}}^{n}$ обслуживает меру $\mathbf{Q}^{n}$, нам потребуется описание семимартингала $X$ относительно $\mathbf{Q}^{n}$.

Теорема A.1 (см. приложение А) дает описание семимартингала $\left(X_{t}, \mathbf{Q}^{n}\right)_{t \in[0, T]}$ :

$$
\begin{aligned}
X_{t \wedge \tau_{n}}=X_{0}+\int_{0}^{t} & I_{\left\{s \leqslant \tau_{n}\right\}}\left[a_{s}\left(X_{s-}\right)+b_{s}\left(X_{s-}\right) \sigma_{s}\left(X_{s-}\right)\right. \\
& \left.+\int_{\mathbf{R}} h_{s}\left(X_{s-}, z\right) \varphi_{s}\left(X_{s-}, z\right) K(d z)\right] d s+\widetilde{\mathscr{M}}_{t}^{c, n}+\widetilde{\mathscr{M}}_{t}^{d, n}
\end{aligned}
$$

с непрерывным и чисто разрывным квадратично интегрируемыми мартингалами $\widetilde{\mathscr{M}}_{t}^{c, n}$ и $\widetilde{\mathscr{M}}_{t}^{d, n}$ соответственно. Их предсказуемые квадратические вариации задаются формулами:

$$
\begin{aligned}
\left\langle\widetilde{\mathscr{M}}^{c, n}\right\rangle_{t} & =\int_{0}^{t} I_{\left\{s \leqslant \tau_{n}\right\}} b_{s}^{2}\left(X_{s-}\right) d s \\
\left\langle\widetilde{\mathscr{M}}^{d, n}\right\rangle_{t} & =\int_{0}^{t} \int_{\mathbf{R}} I_{\left\{s \leqslant \tau_{n}\right\}} h_{s}^{2}\left(X_{s-}, z\right)\left[1+\varphi_{s}\left(X_{s-}, z\right)\right] K(d z) d s .
\end{aligned}
$$

Применяя формулу Ито, получаем

$$
\begin{aligned}
X_{t \wedge \tau_{n}}^{2}= & X_{0}^{2}+\int_{0}^{t} I_{\left\{s \leqslant \tau_{n}\right\}} \cdot 2 X_{s-}\left[a_{s}\left(X_{s-}\right)+b_{s}\left(X_{s-}\right) \sigma_{s}\left(X_{s-}\right)\right. \\
& \left.+\int_{\mathbf{R}} h_{s}\left(X_{s-}, z\right) \varphi_{s}\left(X_{s-}, z\right) K(d z)\right] d s+\left\langle\widetilde{\mathscr{M}}^{c, n}\right\rangle_{t}+\left[\widetilde{\mathscr{M}}^{d, n}, \widetilde{\mathscr{M}}^{d, n}\right]_{t} \\
& +\int_{0}^{t} I_{\left\{s \leqslant \tau_{n}\right\}} \cdot 2 X_{s-} d \widetilde{\mathscr{M}}_{s}^{c, n}+\int_{0}^{t} I_{\left\{s \leqslant \tau_{n}\right\}} \cdot 2 X_{s-} d \widetilde{\mathscr{M}}_{s}^{d, n},
\end{aligned}
$$

где $\left[\mathscr{M}^{d, n}, \mathscr{M}^{d, n}\right]_{t}$ - квадратическая вариация мартингала $\mathscr{M}_{t}^{d, n}$. Отсюда

$$
\begin{aligned}
\widetilde{\mathbf{E}}^{n} X_{t \wedge \tau_{n}}^{2}= & \widetilde{\mathbf{E}}^{n} X_{0}^{2}+\int_{0}^{t} \widetilde{\mathbf{E}}^{n} I_{\left\{s \leqslant \tau_{n}\right\}} \cdot 2 X_{s-}\left[a_{s}\left(X_{s-}\right)+b_{s}\left(X_{s-}\right) \sigma_{s}\left(X_{s-}\right)\right. \\
& \left.+\int_{\mathbf{R}} h_{s}\left(X_{s-}, z\right) \varphi_{s}\left(X_{s-}, z\right) K(d z)\right] d s \\
+ & \widetilde{\mathbf{E}}^{n}\left\langle\widetilde{\mathscr{M}}^{c, n}\right\rangle_{t}+\widetilde{\mathbf{E}}^{n}\left\langle\widetilde{\mathscr{M}}^{d, n}\right\rangle_{t} .
\end{aligned}
$$

Ввиду

$$
\begin{aligned}
\widetilde{\mathbf{E}}^{n}\left\langle\widetilde{\mathscr{M}}^{c, n}\right\rangle_{t} & =\widetilde{\mathbf{E}}^{n} \int_{0}^{t} I_{\left\{s \leqslant \tau_{n}\right\}} b_{s}^{2}\left(X_{s-}\right) d s \\
\widetilde{\mathbf{E}}^{n}\left\langle\widetilde{\mathscr{M}}^{d, n}\right\rangle_{t} & =\widetilde{\mathbf{E}}^{n} \int_{0}^{t} \int_{\mathbf{R}} I_{\left\{s \leqslant \tau_{n}\right\}} h_{s}^{2}\left(X_{s-}, z\right)\left[1+\varphi_{s}\left(X_{s-}, z\right)\right] K(d z) d s
\end{aligned}
$$


выражение (6.4) можно представить следующим образом:

$$
\widetilde{\mathbf{E}}^{n} X_{t \wedge \tau_{n}}^{2}=\widetilde{\mathbf{E}}^{n} X_{0}^{2}+\widetilde{\mathbf{E}}^{n} \int_{0}^{t} I_{\left\{s \leqslant \tau_{n}\right\}} \mathfrak{L}_{s}\left(X_{s-}\right) d s,
$$

где $\mathfrak{L}_{s}\left(X_{s-}\right)$ определено в $(4.2)$, т.е. мы получаем неравенство Гронуолла-Беллмана: $\widetilde{\mathbf{E}}^{n} X_{t \wedge \tau_{n}}^{2} \leqslant \mathrm{r} \int_{0}^{t}\left[1+\widetilde{\mathbf{E}}^{n} X_{s \wedge \tau_{n}}^{2} d s\right]$, которое влечет за собой требуемое неравенство $\sup _{n} \int_{0}^{T} \widetilde{\mathbf{E}}^{n} X_{s \wedge \tau_{n}}^{2} d s \leqslant e^{\mathrm{r} T}-1$. Теорема 4.1 доказана.

6.3. Доказательство теоремы 5.1. Пусть момент остановки $\tau_{n}$ (см. (6.1)) согласован с теоремой 5.1. Тогда в соответствии с леммой 6.1 достаточно проверить условие равномерной интегрируемости последовательности случайных величин $\mathfrak{z}_{T \wedge \tau_{n}}, n \geqslant 1$ :

$$
\sup _{n} \widetilde{\mathbf{E}}^{n} \int_{0}^{T} \sup _{s^{\prime} \leqslant s} X_{s^{\prime} \wedge \tau_{n}}^{2} d s<\infty
$$

Поскольку $\mathbf{E}_{\mathfrak{z}_{T \wedge \tau_{n}}}=1$, вероятностная мера $\mathbf{Q}^{n}$ определена так же, как в доказательстве теоремы 4.1: $d \mathbf{Q}^{n}=\mathfrak{z}_{T \wedge \tau_{n}} d \mathbf{P}$. Поэтому, так же как в доказательстве теоремы 4.1, воспользуемся теоремой А.1, для того чтобы получить семимартингальное разложение процесса $\left(X_{t \wedge \tau_{n}}, \mathbf{Q}^{n}\right)_{t \in[0, T]}$ :

$$
\begin{aligned}
X_{t \wedge \tau_{n}}=X_{0}+\int_{0}^{t} I_{\left\{s \leqslant \tau_{n}\right\}}\left[a_{s}(X)+b_{s}(X) \sigma_{s}(X),\right. \\
\left.\quad+\int_{\mathbf{R}} h_{s}(X, z) \varphi_{s}(X, z) K(d z)\right] d s+\widetilde{\mathscr{M}}_{t}^{c, n}+\widetilde{\mathscr{M}}_{t}^{d, n},
\end{aligned}
$$

где $\widetilde{\mathscr{M}}_{t}^{c, n}$ и $\widetilde{\mathscr{M}}_{t}^{d, n}$ - непрерывный и чисто разрывный квадратично интегрируемые мартингалы с

$$
\begin{aligned}
\left\langle\widetilde{\mathscr{M}}^{c, n}\right\rangle_{t} & =\int_{0}^{t} I_{\left\{s \leqslant \tau_{n}\right\}} b_{s}^{2}(X) d s, \\
\left\langle\widetilde{\mathscr{M}}^{d, n}\right\rangle_{t} & =\int_{0}^{t} \int_{\mathbf{R}} I_{\left\{s \leqslant \tau_{n}\right\}} h_{s}^{2}(X, z)\left[1+\varphi_{s}(X, z)\right] K(d z) d s .
\end{aligned}
$$

Семимартингал, определенный в (6.5), позволяет получить оценку

$$
\begin{aligned}
\widetilde{\mathbf{E}}^{n} \sup _{t^{\prime} \leqslant t}\left|X_{t^{\prime} \wedge \tau_{n}}\right|^{2} \leqslant & 4\left[\widetilde{\mathbf{E}}^{n} X_{0}^{2}+\widetilde{\mathbf{E}}^{n} \sup _{t^{\prime} \leqslant t}\left|\mathscr{M}_{t^{\prime}}^{c, n}\right|^{2}+\widetilde{\mathbf{E}}^{n} \sup _{t^{\prime} \leqslant t}\left|\mathscr{M}_{t^{\prime}}^{d, n}\right|^{2}\right. \\
& +\widetilde{\mathbf{E}}^{n}\left(\int_{0}^{t} I_{\left\{s \leqslant \tau_{n}\right\}} \mid a_{s}(X)+b_{s}(X) \sigma_{s}(X)\right. \\
& \left.\left.\quad+\int_{\mathbf{R}} h_{s}(X, z) \varphi_{s}(X, z) K(d z) \mid d s\right)^{2}\right] .
\end{aligned}
$$


Оценим каждый член в правой части этого неравенства (последнее математическое ожидание в квадратных скобках обозначим $J_{t}$ ):

$4 \widetilde{\mathbf{E}}^{n} X_{0}^{2} \leqslant \mathrm{r} \quad$ (по условию теоремы);

$4 \widetilde{\mathbf{E}}^{n} \sup _{t^{\prime} \leqslant t}\left|\mathscr{M}_{t^{\prime}}^{c, n}\right|^{2} \leqslant 16 \widetilde{\mathbf{E}}^{n}\left\langle\mathscr{M}^{c, n}\right\rangle_{t}=16 \widetilde{\mathbf{E}}^{n} \int_{0}^{t} I_{\left\{s \leqslant \tau_{n}\right\}} b_{s}^{2}(X) d s$

(максимальное неравенство Дуба);

$4 \widetilde{\mathbf{E}}^{n} \sup _{t^{\prime} \leqslant t}\left|\mathscr{M}_{t^{\prime}}^{d, n}\right|^{2} \leqslant 16 \widetilde{\mathbf{E}}^{n}\left\langle\mathscr{M}^{d, n}\right\rangle_{t}$

$$
=16 \widetilde{\mathbf{E}}^{n} \int_{0}^{t} \int_{\mathbf{R}} I_{\left\{s \leqslant \tau_{n}\right\}} h_{s}^{2}(X, z)\left[1+\varphi_{s}(X, z)\right] K(d z) d s
$$

(максимальное неравенство Дуба);

$$
\begin{aligned}
& 4 J_{t} \leqslant 4 t \mathbf{E} \int_{0}^{t} I_{\left\{s \leqslant \tau_{n}\right\}}\left|a_{s}(X)+b_{s}(X) \sigma_{s}(X)+\int_{\mathbf{R}} h_{s}(X, z) \varphi_{s}(X, z) K(d z)\right|^{2} d s \\
& \leqslant 12 t \mathbf{E} \int_{0}^{t} I_{\left\{s \leqslant \tau_{n}\right\}}\left[a_{s}^{2}(X)+b_{s}^{2}(X) \sigma_{s}^{2}(X)\right. \\
& \left.+\left(\int_{\mathbf{R}} h_{s}(X, z) \varphi_{s}(X, z) K(d z)\right)^{2}\right] d s \\
& \leqslant 12 t \mathbf{E} \int_{0}^{t} I_{\left\{s \leqslant \tau_{n}\right\}}\left[a_{s}^{2}(X)+b_{s}^{2}(X) \sigma_{s}^{2}(X)\right. \\
& \left.+\int_{\mathbf{R}} h_{s}^{2}(X, z) K(d z) \int_{\mathbf{R}} \varphi_{s}(X, z) K(d z)\right] d s
\end{aligned}
$$

(неравенство Коши-Шварца-Буняковского).

Вся совокупность представленных выше оценок влечет за собой неравенство $\widetilde{\mathbf{E}}^{n} \sup _{t^{\prime} \leqslant t}\left|X_{t^{\prime} \wedge \tau_{n}}\right|^{2} \leqslant \mathrm{r}+\int_{0}^{t} \widetilde{\mathbf{E}}^{n} \sup _{t^{\prime} \leqslant t} I_{\left\{s \leqslant \tau_{n}\right\}} \mathfrak{L}_{s}(X) d s$, где оператор $\mathfrak{\sim}_{s}(X)$ определяется с помощью формулы (5.1), т.е. $\widetilde{\mathbf{E}}^{n} \mathfrak{L}_{s \wedge \tau_{n}}(X) \leqslant \mathrm{r}[1+$ $\left.\widetilde{\mathbf{E}}^{n} \sup _{s^{\prime} \leqslant s}\left|X_{s^{\prime} \wedge \tau_{n}}\right|^{2}\right]$. В результате получаем неравенство Гронуолла-Беллмана:

$$
\widetilde{\mathbf{E}}^{n} \sup _{t^{\prime} \leqslant t}\left|X_{t^{\prime} \wedge \tau_{n}}\right|^{2} \leqslant \mathbf{r}\left[1+\int_{0}^{t} \widetilde{\mathbf{E}}^{n} \sup _{s^{\prime} \leqslant s}\left|X_{s^{\prime} \wedge \tau_{n}}\right|^{2} d s\right],
$$

и, значит, требуемая оценка $\sup _{n} \int_{0}^{T} \widetilde{\mathbf{E}}^{n} \sup _{s^{\prime} \leqslant s}\left|X_{s^{\prime} \wedge \tau_{n}}\right|^{2} d s \leqslant e^{\mathrm{r} T}-1$ имеет место. Теорема 5.1 доказана.

7. Пример марковского процесса $X$, допускающего взрыв. В этом разделе мы рассмотрим конкретную модель (по поводу других примеров см. [1]). Эта модель

$$
\begin{aligned}
X_{t}= & X_{0}+\int_{0}^{t} a_{s}\left(X_{s-}\right) d s+\int_{0}^{t} b_{s}\left(X_{s-}\right) d B_{s} \\
& +\int_{0}^{t} \int_{\mathbf{R}} h_{s}\left(X_{s-}, z\right)[\mu(d s, d z)-d s K(d z)] \\
\mathfrak{z}_{t}= & 1+\int_{0}^{t} \mathfrak{z}_{s-}\left[\sigma_{s}\left(X_{s-}\right) d B_{s}+\int_{\mathbf{R}} \varphi_{s}\left(X_{s-}, z\right)[\mu(d s, d z)-d s K(d z)]\right]
\end{aligned}
$$


обобщает модель Миятовича-Урусова [29], поскольку она содержит чисто разрывные мартингалы.

Мы фиксируем следующие условия:

1. $a_{s}\left(x_{s-}\right) \geqslant\left|x_{s-}\right|^{\alpha}, \alpha>3 ; \quad$ 2. $b_{s}^{2}\left(x_{s-}\right) \leqslant \begin{cases}\mathrm{r}, & \alpha \in(3,4), \\ \mathrm{r}\left[1+x_{s-}^{2}\right], & \alpha>4 ;\end{cases}$

3. $h_{s}\left(x_{s-}, z\right) \equiv z ; \quad$ 4. $\sigma_{s}^{2}\left(x_{s-}\right) \leqslant \mathrm{r}\left[1+x_{s-}^{2}\right] ; \quad$ 5. $\varphi_{s}\left(x_{s-}, z\right) \equiv|z|$;

6. $\int_{\mathbf{R}}\left[z^{2}+|z|^{3}\right] K(d z)<\infty ; \quad$ 7. $0<X_{0} \leqslant \mathrm{r}$.

Условие

$$
\sigma_{s}^{2}\left(x_{s-}\right)+\int_{\mathbf{R}} \varphi_{s}^{2}\left(x_{s-}, z\right) K(d z) \leqslant\left[1+x_{s-}^{2}\right], \quad s \in[0, T],
$$

предполагается выполненным, в то время как условия, связанные с операторами $L_{s}\left(x_{s-}\right)$ и $\mathfrak{L}_{s}\left(x_{s-}\right)$, не выполняются. Поэтому взрыв процесса $X_{t}$ по направлению к $+\infty$ не исключается. В случае взрыва последовательность $\tau_{n}=\inf \left\{t:\left(\mathfrak{z}_{t} \vee X_{t}^{2}\right) \geqslant n\right\}, n \geqslant 1$, имеет предел $\tau \leqslant \infty$ с $\mathbf{P}(\tau<\infty)>0$. Следовательно, в случае взрыва можно ожидать только равенство $\mathbf{E}_{\mathfrak{z}_{T \wedge \tau}}=1$. Несмотря на то что возможность взрыва не нарушает утверждения леммы 6.1 , проверка условия $\sup _{n} \widetilde{\mathbf{E}}^{n} \int_{0}^{T} X_{s \wedge \tau_{n}}^{2} d s<\infty$ этой леммы вряд ли может быть эффективно реализована. Поэтому вместо этого условия мы используем следующее:

$$
\sup _{n} \widetilde{\mathbf{E}}^{n} \int_{0}^{T} \frac{\left|X_{s \wedge \tau_{n}}\right|^{\alpha}}{1+\left|X_{s \wedge \tau_{n}}\right|^{\alpha-2}} d s<\infty,
$$

тем более, что при $\alpha>3$

$$
\sup _{n} \widetilde{\mathbf{E}}^{n} \int_{0}^{T} \frac{\left|X_{s \wedge \tau_{n}}\right|^{\alpha}}{1+\left|X_{s \wedge \tau_{n}}\right|^{\alpha-2}} d s<\infty \Rightarrow \sup _{n} \widetilde{\mathbf{E}}^{n} \int_{0}^{T} X_{s \wedge \tau_{n}}^{2} d s<\infty .
$$

Выберем функцию

$$
g_{\alpha}(u)=\int_{0}^{u} \frac{1}{1+|y|^{\alpha-2}} d y, \quad u \in \mathbf{R} .
$$

Поскольку ее производные имеют вид

$$
g_{\alpha}^{\prime}(u)=\frac{1}{1+|u|^{\alpha-2}} \quad \text { и } \quad g_{\alpha}^{\prime \prime}(u)=-\frac{(\alpha-2)|u|^{\alpha-3} \operatorname{sign} u}{\left[1+|u|^{\alpha-2}\right]^{2}},
$$

то $a_{s}\left(x_{s-}\right) \geqslant\left|x_{s-}\right|^{\alpha}$ и $\left|g_{\alpha}^{\prime \prime}\left(x_{s-}\right)\right| \leqslant r$. Более того,

$$
g_{\alpha}^{\prime}\left(x_{s-}\right) a_{s}\left(x_{s-}\right) \geqslant \frac{\left|x_{s-}\right|^{\alpha}}{1+\left|x_{s-}\right|^{\alpha-2}}
$$


где правая часть есть не что иное, как подынтегральная функция в (7.1).

Процесс $X_{t \wedge \tau_{n}}$ относительно новой меры $\mathbf{Q}^{n}$ не взрывается ни при каком $n$. Поэтому по теореме А.1,

$$
\begin{aligned}
X_{t \wedge \tau_{n}}=X_{0}+\int_{0}^{t} I_{\left\{s \leqslant \tau_{n}\right\}}\left[a_{s}\left(X_{s-}\right)\right. & +b_{s}\left(X_{s-}\right) \sigma_{s}\left(X_{s-}\right) \\
& \left.+\int_{\mathbf{R}} z|z| K(d z)\right] d s+\widetilde{\mathscr{M}}_{t}^{c, n}+\widetilde{\mathscr{M}}_{t}^{d, n},
\end{aligned}
$$

где $\widetilde{\mathscr{M}}_{t}^{c, n}$ и $\widetilde{\mathscr{M}}_{t}^{d, n}$ - непрерывный и чисто разрывный квадратично интегрируемые мартингалы с предсказуемыми характеристиками

$$
\begin{aligned}
\left\langle\widetilde{\mathscr{M}}^{c, n}\right\rangle_{t} & =\int_{0}^{t} I_{\left\{s \leqslant \tau_{n}\right\}} b_{s}^{2}\left(X_{s-}\right) d s \\
\left\langle\widetilde{\mathscr{M}}^{d, n}\right\rangle_{t} & =\int_{0}^{t} \int_{\mathbf{R}} I_{\left\{s \leqslant \tau_{n}\right\}}\left[z^{2}+|z|^{3}\right] K(d z) d s .
\end{aligned}
$$

Теперь применим формулу Ито к $g_{\alpha}\left(X_{t \wedge \tau_{n}}\right)$ :

$$
\begin{aligned}
g_{\alpha}( & \left.X_{T \wedge \tau_{n}}\right)=g_{\alpha}\left(X_{0}\right) \\
& +\int_{0}^{T} I_{\left\{s \leqslant \tau_{n}\right\}} g_{\alpha}^{\prime}\left(X_{s-}\right)\left[a_{s}\left(X_{s-}\right)+\sigma_{s}\left(X_{s-}\right) b_{s}\left(X_{s}\right)+\int_{\mathbf{R}}|z| z K(d z)\right] d s \\
& +\int_{0}^{t} I_{\left\{s \leqslant \tau_{n}\right\}} g_{\alpha}^{\prime}\left(X_{s-}\right) d \widetilde{\mathscr{M}}_{s}^{c, n}+\int_{0}^{t} I_{\left\{s \leqslant \tau_{n}\right\}} g_{\alpha}^{\prime}\left(X_{s-}\right) d \widetilde{\mathscr{M}}_{s}^{d, n} \\
& +\frac{1}{2} \int_{0}^{t} I_{\left\{s \leqslant \tau_{n}\right\}} g_{\alpha}^{\prime \prime}\left(X_{s-}\right) d\left\langle\widetilde{\mathscr{M}}^{c, n}\right\rangle_{s} \\
& +\int_{0}^{t} \int_{\mathbf{R}} I_{\left\{s \leqslant \tau_{n}\right\}}\left[g_{\alpha}\left(X_{s-}+z\right)-g_{\alpha}\left(X_{s-}\right)-g_{\alpha}^{\prime}\left(X_{s-}\right) z\right] \mu(d s, d z) .
\end{aligned}
$$

Тогда

$$
\begin{aligned}
\widetilde{\mathbf{E}}^{n} g_{\alpha} & \left(X_{T \wedge \tau_{n}}\right)=\widetilde{\mathbf{E}}^{n} g_{\alpha}\left(X_{0}\right)+\widetilde{\mathbf{E}}^{n} \int_{0}^{T} I_{\left\{s \leqslant \tau_{n}\right\}} g_{\alpha}^{\prime}\left(X_{s-}\right) a_{s}\left(X_{s}\right) d s \\
& +\widetilde{\mathbf{E}}^{n} \int_{0}^{T} I_{\left\{s \leqslant \tau_{n}\right\}} g_{\alpha}^{\prime}\left(X_{s}\right)\left[\sigma_{s}\left(X_{s-}\right) b_{s}\left(X_{s-}\right)+\int_{\mathbf{R}}|z| z K(d z)\right] d s \\
& +\frac{1}{2} \widetilde{\mathbf{E}}^{n} \int_{0}^{T} I_{\left\{s \leqslant \tau_{n}\right\}} g_{\alpha}^{\prime \prime}\left(X_{s-}\right) b_{s}^{2}\left(X_{s}\right) d s \\
& +\widetilde{\mathbf{E}}^{n} \int_{0}^{T} \int_{\mathbf{R}} I_{\left\{s \leqslant \tau_{n}\right\}}\left[g\left(X_{s-}+z\right)-g\left(X_{s-}\right)-g^{\prime}\left(X_{s-}\right) z\right] \nu^{n}(d s, d z),
\end{aligned}
$$

где $\nu^{n}(d s, d z)=I_{\left\{s \leqslant \tau_{n}\right\}}[1+|z|] d s K(d z)$ является $\widetilde{\mathbf{Q}}^{n}$-компенсатором целочисленной случайной меры $I_{\left\{s \leqslant \tau_{n}\right\}} \mu(d s, d z)$ (см. теорему А.1). Отсюда, с учетом (7.2), выводим следующую верхнюю границу в виде суммы 
случайных величин:

$$
\begin{aligned}
& \widetilde{\mathbf{E}}^{n} \int_{0}^{T} I_{\left\{s \leqslant \tau_{n}\right\}} \frac{\left|X_{s-}\right|^{\alpha}}{1+\left|X_{s-}\right|^{\alpha-2}} d s \leqslant \widetilde{\mathbf{E}}^{n} \int_{0}^{T} I_{\left\{s \leqslant \tau_{n}\right\}} g_{\alpha}^{\prime}\left(X_{s-}\right) a_{s}\left(X_{s-}\right) d s \\
& \leqslant \widetilde{\mathbf{E}}^{n} g_{\alpha}\left(X_{T \wedge \tau_{n}}\right)+\widetilde{\mathbf{E}}^{n} \int_{0}^{T} I_{\left\{s \leqslant \tau_{n}\right\}} g_{\alpha}^{\prime}\left(X_{s-}\right)\left|\sigma_{s}(X) b_{s}(X-)\right| d s \\
&+\widetilde{\mathbf{E}}^{n} \int_{0}^{T} \int_{\mathbf{R}} I_{\left\{s \leqslant \tau_{n}\right\}} g^{\prime}\left(X_{s-}\right) z^{2} K(d z) d s \\
&+\frac{1}{2} \widetilde{\mathbf{E}}^{n} \int_{0}^{T} I_{\left\{s \leqslant \tau_{n}\right\}}\left|g_{\alpha}^{\prime \prime}\left(X_{s-}\right)\right| b_{s}^{2}\left(X_{s-}\right) d s \\
&+\widetilde{\mathbf{E}}^{n} \int_{0}^{T} \int_{\mathbf{R}} I_{\left\{s \leqslant \tau_{n}\right\}} \mid g_{\alpha}\left(X_{s-}+z\right)-g_{\alpha}\left(X_{s-}\right)-g_{\alpha}^{\prime}\left(X_{s-}\right) z \\
& \mid[1+|z|] K(d z) d s .
\end{aligned}
$$

Абсолютное значение каждого слагаемого из этой суммы ограничено константой, не зависящей от $n$ :

$$
\begin{aligned}
& g_{\alpha}\left(X_{T \wedge \tau_{n}}\right) \leqslant \text { const, } \quad \alpha>3 ; \\
& g_{\alpha}^{\prime}\left(X_{\left(t \wedge \tau_{n}\right)-}\right)\left|\sigma_{t}\left(X_{\left(t \wedge \tau_{n}\right)-}\right) b_{t}\left(X_{\left(t \wedge \tau_{n}\right)-}\right)\right| \\
& \leqslant \begin{cases}\frac{\sqrt{1+X_{\left(t \wedge \tau_{n}\right)-}^{2}}}{1+\left|X_{\left(t \wedge \tau_{n}\right)-}^{\alpha-2}\right|} \leqslant \text { const, } & \alpha \in(3,4), \\
\frac{1+X_{\left(t \wedge \tau_{n}\right)-}^{2}}{1+\left|X_{\left(t \wedge \tau_{n}\right)-}^{2}\right|^{\alpha-2}} \leqslant \mathrm{const}, & \alpha \geqslant 4 ;\end{cases} \\
& \left|g_{\alpha}^{\prime}\left(X_{\left(t \wedge \tau_{n}\right)-}\right) \int_{\mathbf{R}} z^{2} K(d z)\right| \leqslant \text { const; } \\
& \int_{0}^{T}\left|g_{\alpha}^{\prime \prime}\left(X_{\left(s \wedge \tau_{n}\right)-}\right)\right| b_{s}^{2}\left(X_{\left(s \wedge \tau_{n}\right)-}\right) d s \\
& \leqslant \int_{0}^{T} \frac{(\alpha-2)\left|X_{\left(s \wedge \tau_{n}\right)-}\right|^{\alpha-3}}{\left[1+\left|X_{\left(s \wedge \tau_{n}\right)-}\right|^{\alpha-2}\right]^{2}}\left[1+X_{\left(s \wedge \tau_{n}\right)-}^{2}\right] \int_{\mathbf{R}} z^{2} K(d z) d s \leqslant \mathrm{const} ; \\
& \int_{0}^{T} \int_{\mathbf{R}}\left|g_{\alpha}\left(X_{s \wedge \tau_{n}}+z\right)-g_{\alpha}\left(X_{s \wedge \tau_{n}}\right)-g_{\alpha}^{\prime}\left(X_{\left(s \wedge \tau_{n}\right)-}\right) z\right|[1+|z|] K(d z) d s \\
& \leqslant r T \int_{\mathbf{R}}\left[z^{2}+|z|^{3}\right] K(d z) .
\end{aligned}
$$

Таким образом (7.1) имеет место.

\section{8. Дополнения.}

8.1. $\mathbf{E}_{\mathfrak{z}_{T}}<1$ может нарушить условие Бенеша. Пусть $X_{t}-$ процесс Бесселя: $X_{t}=1+\int_{0}^{t}\left(1 / X_{s}\right) d s+B_{t}$. Процесс $X_{t}$ положителен и, значит, формула Ито применима к $\ln x$ :

$$
\ln X_{t}=-\int_{0}^{t} \frac{1}{2 X_{s}^{2}} d s+\int_{0}^{t} \frac{d B_{s}}{X_{s}} .
$$


Пусть $M_{t}=\int_{0}^{t} X_{s} d B_{s}$ и

$$
\mathfrak{z}_{t}=1-\int_{0}^{t} \mathfrak{z}_{s} \frac{d B_{s}}{X_{s}}
$$

Предположим, что найдется момент времени $T>0$ такой, что $\mathbf{E}_{\mathfrak{z}_{T}}=1$. Тогда существует вероятностная мера $\mathbf{Q} \ll \mathbf{P}$ с плотностью $d \mathbf{Q} / d \mathbf{P}=$ $\mathfrak{z}_{T}$, а теорема Гирсанова позволяет представить процесс $X_{t}$ (относительно меры $\mathbf{Q} x)$ в виде

$$
X_{t}=1+\widetilde{B}_{t}
$$

с Q-броуновским движением $\widetilde{B}_{t}$. Значит, $X_{t}$ - гауссовский процесс, который не может быть только положительным. Поэтому $\mathbf{E}_{\mathfrak{z}_{T}} \neq 1$, т.е. $\mathbf{E}_{\mathfrak{z}_{T}}<1$. С другой стороны, $\sigma^{2}(y)=1 / y^{2} \nless \mathbf{r}\left[1+y^{2}\right]$ невозможно.

8.2. $X$ - векторная диффузия. Поскольку мы имеем дело с диффузионным процессом, мы будем использовать обозначения $x_{s}, X_{s}$ вместо $x_{s-}, X_{s-}$. Пусть

$$
X_{t}=X_{0}+\int_{0}^{t} a_{s}\left(X_{s}\right) d s+\int_{0}^{t} b_{s}\left(X_{s}\right) d B_{s},
$$

где $a_{s}\left(x_{s}\right)$ - матричная функция, $b_{s}\left(x_{s}\right)$ - векторная функция (столбец), $B_{t}$ - броуновский векторный процесс (столбец) с независимыми компонентами, являющимися стандартными броуновскими движениями.

Условие Бенеша естественным образом адаптируется к векторному случаю. Норму в $\mathbf{L}^{2}$ и скалярное произведение векторов обозначим $\|\cdot\|$ и $\langle\langle\cdot\rangle\rangle$ соответственно. Символ транспонирования матриц обозначим * . Пусть $\sigma_{s}\left(x_{s}\right)$ - векторная функция (строка) такая, что процесс

$$
\mathfrak{z}_{t}=1+\int_{0}^{t} \mathfrak{z}_{s}\left\langle\left\langle\sigma_{s}\left(x_{s}\right), d B_{s}\right\rangle\right\rangle
$$

определен.

Теорема 8.1. Пусть $\|X\|_{0}^{2} \leqslant \mathrm{r} u\left(x_{s}\right)_{s \in \mathbf{R}_{+}} \in \mathbf{C}$. Предположим, ито для любого $\left(x_{s}\right)_{s \in[0, T]} \in \mathbf{C}$ определень

1) $\left\|\sigma_{s}\left(x_{s}\right)\right\|^{2}$

2) $L_{s}\left(x_{s}\right)=2\left\langle\left\langle x_{s}, a_{s}(x)\right\rangle\right\rangle+\operatorname{trace}\left[b_{s}^{*}\left(x_{s}\right) b_{s}\left(x_{s}\right)\right]$,

3) $\mathfrak{L}_{s}\left(x_{s}\right)=2\left\langle\left\langle x_{s},\left[a_{s}\left(x_{s}\right)+b_{s}^{*}\left(x_{s}\right) \sigma_{s}\left(x_{s}\right)\right]\right\rangle\right\rangle+\operatorname{trace}\left[b_{s}^{*}\left(x_{s}\right) b_{s}\left(x_{s}\right)\right]$.

Если для любого $s \leqslant T$

$$
\left\|\sigma_{s}\left(x_{s}\right)\right\|^{2}+L_{s}\left(x_{s}\right)+\mathfrak{L}_{s}\left(x_{s}\right) \leqslant \mathrm{r}\left[1+\left\|x_{s}\right\|^{2}\right],
$$

$m о \mathbf{E}_{\mathfrak{z}_{T}}=1$ для любого $T>0$.

Доказательство этой теоремы такое же, как в скалярном случае, и поэтому опускается. 
П р и м е р 8.1. Пусть $X_{t}=X_{0}+\int_{0}^{t} a\left(X_{s}\right) d s+\int_{0}^{t} b\left(X_{s}\right) d B_{s}$ и

$$
\mathfrak{z}_{t}=1+\int_{0}^{t} \mathfrak{z}_{s}\left[\theta\left(X_{s}\right) d W_{s}+\sigma\left(X_{s}\right) d B_{s}\right]
$$

где $W_{t}$ - винеровский процесс, не зависящий от $B_{t}$. Несмотря на то что $X_{t}$ - скалярный процесс, для проверки условия $\mathbf{E}_{\mathfrak{z}_{T}}=1$ удобно применить теорему 8.1. Пишем

$$
\begin{gathered}
\left(\begin{array}{c}
X_{t} \\
0
\end{array}\right)=\left(\begin{array}{c}
X_{0} \\
0
\end{array}\right)+\int_{0}^{t}\left(\begin{array}{c}
a\left(X_{s}\right) \\
0
\end{array}\right) d s+\int_{0}^{t}\left(\begin{array}{cc}
b\left(X_{s}\right) & 0 \\
0 & 0
\end{array}\right)\left(\begin{array}{c}
d B_{s} \\
d W_{s}
\end{array}\right), \\
\mathfrak{z}_{t}=1+\int_{0}^{t} \mathfrak{z}_{s}\left[\theta\left(X_{s}\right) d W_{s}+\sigma\left(X_{s}\right) d B_{s}\right] .
\end{gathered}
$$

Если

$$
\begin{gathered}
X_{0}^{2} \leqslant \mathrm{r}, \quad \sigma^{2}(x)+\theta^{2}(x) \leqslant \mathrm{r}\left[1+x^{2}\right], \quad 2 x a(x)+b^{2}(x) \leqslant \mathrm{r}\left[1+x^{2}\right], \\
2 x[a(x)+b(x) \sigma(x)]+b^{2}(x) \leqslant \mathrm{r}\left[1+x^{2}\right],
\end{gathered}
$$

то $\mathbf{E}_{\mathfrak{z}_{T}}=1$ для любого $T>0$.

Предположим, что $\sigma^{2}(x) \equiv 0$. В этом случае $X_{t}$ и $W_{t}-$ независимые процессы и имеет место хорошо известный результат

$$
\mathbf{E}_{\mathfrak{z}_{T}}=\mathbf{E} \exp \left(\int_{0}^{T} \theta\left(X_{s}\right) d W_{s}-\frac{1}{2} \int_{0}^{T} \theta^{2}\left(X_{s}\right) d s\right)=1 .
$$

\section{3. Модель Хитсуды. Нелинейная версия. Пусть}

$$
X_{t}=\int_{0}^{t} \int_{0}^{s} l(s, u) d B_{u} d s+B_{t} \quad \text { и } \quad \mathfrak{z}_{t}=1+\int_{0}^{t} \mathfrak{z}_{s} \sigma\left(X_{s}\right) d B_{s}
$$

где $l(t, s)$ - ядро Вольтерра, $\int_{0}^{t} \int_{0}^{s} l^{2}(s, u) d u d s<\infty$ и $\sigma(x)$ - нелинейная функция. В случае $\sigma(x)=x$ условие $\iint_{[0, T]^{2}} l^{2}(s, u) d u d s<\infty$ обеспечивает $\mathbf{E}_{\mathfrak{z}_{T}}=1$ (см. [11]).

Если $\sigma(x)$ - нелинейная (и, возможно, разрывная) функция, то, комбинируя подход Хитсуды с условием Бенеша, можно получить следующий результат.

Теорема 8.2. Если выполнень условия

$$
\text { 1) } \left.\sigma^{2}(x) \leqslant r\left[1+x^{2}\right], s \in[0, T], \quad 2\right) \quad \iint_{[0, T]^{2}} l^{2}(t, s) d t d s<\infty,
$$

$m o \mathbf{E}_{\mathfrak{z}_{T}}=1$.

Д ок а з а т е л ь с т о. Формально эта модель не согласована с условиями теорем 4.1 и 5.1. Тем не менее выбор $\tau_{n}=\inf \left\{t:\left(\mathfrak{z}_{t} \vee X_{t}^{2}\right) \geqslant n\right\}$ и условие

$$
\sup _{n} \widetilde{\mathbf{E}}^{n} \int_{0}^{T} X_{t \wedge \tau_{n}}^{2} d t<\infty
$$


(см. лемму 6.1) обеспечивают $\mathbf{E}_{\mathfrak{z}_{T}}=1$.

Условие 1) обеспечивает равенство $\mathbf{E}_{\mathfrak{z}_{\text {} \wedge \tau_{n}}}=1$, т.е. гарантирует существование вероятностной меры $\mathbf{Q}^{n}$. Тогда, по теореме Гирсанова, случайный процесс $\left(B_{t \wedge \tau_{n}}, \mathbf{Q}^{n}\right)$ допускает представление

$$
B_{t \wedge \tau_{n}}=\int_{0}^{t} I_{\left\{s \leqslant \tau_{n}\right\}} \sigma\left(X_{s}\right) d s+\widetilde{B}_{t}^{n}
$$

в котором $\widetilde{B}_{t}^{n}$ является $\mathbf{Q}^{n}$-броуновским движением, остановленным в момент времени $\tau_{n}$. Теперь случайный процесс $\left(X_{t \wedge \tau_{n}}, \mathbf{Q}^{n}\right)_{t \in[0, T]}-$ семимартингал:

$$
\begin{aligned}
X_{t \wedge \tau_{n}}= & \int_{0}^{t} I_{\left\{s \wedge \tau_{n}\right\}} \int_{0}^{s} l(s, u) d \widetilde{B}_{u}^{n} d s+\int_{0}^{t} I_{\left\{s \wedge \tau_{n}\right\}} \int_{0}^{s} l(s, u) \sigma\left(X_{u}\right) d u d s \\
& +\int_{0}^{t} I_{\left\{s \leqslant \tau_{n}\right\}} \sigma_{s}(X) d s+\widetilde{B}_{t}^{n} .
\end{aligned}
$$

Мы будем использовать это представление для доказательства (8.1). Нам потребуется оценить $\widetilde{\mathbf{E}}^{n} X_{t \wedge \tau_{n}}^{2}$. С этой целью мы оценим математическое ожидание $\widetilde{\mathbf{E}}^{n}$ квадрата каждого члена в правой части (8.2). Применяя неравенства Коши-Шварца-Буняковского и максимальное неравенство Дуба для квадратично интегрируемого мартингала, а также условие 1) теоремы, получаем следующие оценки.

Первый член:

$$
\begin{gathered}
\widetilde{\mathbf{E}}^{n}\left(\int_{0}^{t^{\prime}} I_{\left\{s \leqslant \tau_{n}\right\}} \int_{0}^{s} l(s, u) d \widetilde{B}_{u}^{n} d s\right)^{2} \leqslant \widetilde{\mathbf{E}}^{n}\left(\int_{0}^{t} \sup _{s \leqslant t}\left|\int_{0}^{s} l(s, u) d \widetilde{B}_{u}^{n}\right| d s\right)^{2} \\
\leqslant t \widetilde{\mathbf{E}}^{n}\left(\int_{0}^{t} \sup _{s \leqslant t}\left|\int_{0}^{s} l(s, u) d \widetilde{B}_{u}^{n}\right|^{2} d s\right) \leqslant 4 t^{2} \iint_{[0, T]^{2}} l^{2}(s, u) d u d s .
\end{gathered}
$$

Второй член:

$$
\begin{aligned}
\widetilde{\mathbf{E}}^{n}\left(\int_{0}^{t} I_{\left\{s \leqslant \tau_{n}\right\}} \int_{0}^{s} l(s, u) \sigma\left(X_{u}\right) d u d s\right)^{2} \\
\quad \leqslant t \int_{0}^{t} \widetilde{\mathbf{E}}^{n} I_{\left\{s \leqslant \tau_{n}\right\}}\left|\int_{0}^{s} l(s, u) \sigma\left(X_{u}\right) d u\right|^{2} d s \\
\quad \leqslant t \int_{0}^{t} \int_{0}^{s} l^{2}(s, u) d u \widetilde{\mathbf{E}}^{n} \int_{0}^{s \wedge \tau_{n}} \sigma^{2}\left(X_{v}\right) d v d s \\
\quad \leqslant t \int_{0}^{t} \int_{0}^{s} l^{2}(s, u) d u \int_{0}^{s} \mathrm{r}\left[1+\widetilde{\mathbf{E}}^{n} X_{v \wedge \tau_{n}}^{2}\right] d v d s .
\end{aligned}
$$

Третий член: $\widetilde{\mathbf{E}}^{n}\left(\int_{0}^{t} I_{\left\{s \leqslant \tau_{n}\right\}} \sigma_{s}(X) d s\right)^{2} \leqslant t \int_{0}^{t} \mathbf{r}\left[1+\widetilde{\mathbf{E}}^{n} X_{v \wedge \tau_{n}}^{2}\right] d v$. Четвертый член: $\widetilde{\mathbf{E}}^{n}\left(\widetilde{B}_{t}^{n}\right)^{2} \leqslant \widetilde{\mathbf{E}}^{n}\left(t \wedge \tau_{n}\right) \leqslant t$. 
Полученные оценки позволяют установить неравенство ГронуоллаБеллмана:

$$
\widetilde{\mathbf{E}}^{n} X_{t \wedge \tau_{n}}^{2} \leqslant \mathrm{r} \iint_{[0, T]^{2}} l^{2}(t, s) d t d s\left[1+\int_{0}^{t} \widetilde{\mathbf{E}}^{n} X_{s \wedge \tau_{n}}^{2} d s\right]
$$

и, ввиду условия 2) теоремы, проверить справедливость (8.1). Теорема 8.2 доказана.

Приложение А. Обобщенная теорема Гирсанова. Пусть $X-$ решение уравнения (3.1) и момент остановки $\tau_{n}$ определен в (6.1). Тогда

$$
\begin{aligned}
X_{t \wedge \tau_{n}}=X_{0}+\int_{0}^{t} I_{\left\{s \leqslant \tau_{n}\right\}} a_{s}(X) d s \\
\quad+\underbrace{\int_{0}^{t} I_{\left\{s \leqslant \tau_{n}\right\}} b_{s}(X) d B_{s}}_{=\mathscr{M}_{t}^{c, n}}+\underbrace{\int_{0}^{t} \int_{\mathbf{R}} I_{\left\{s \leqslant \tau_{n}\right\}} h_{s}(X, z)[\mu(d s, d z)-d s K(d z)]}_{=\mathscr{M}_{t}^{d, n}} .
\end{aligned}
$$

Пусть случайная величина $\mathfrak{z}_{T \wedge \tau_{n}}$ определена в (6.2). Напомним, что $\mathbf{E}_{\mathfrak{z}_{T \wedge \tau_{n}}}=1$, и определим вероятностную меру $\mathbf{Q}^{n}$ с плотностью $d \mathbf{Q}^{n} / d \mathbf{P}=\mathfrak{z}_{T \wedge \tau_{n}}$

Теорема A.1. 1) мера $\nu^{n}(d s, d z)=I_{\left\{s \leqslant \tau_{n}\right\}}\left[1+\varphi\left(s, X_{s-}, z\right)\right] d s K(d z)$ является $\mathbf{Q}^{n}$-компенсатором иелочисленной мерь $I_{\left\{s \leqslant \tau_{n}\right\}} \mu(d s, d z)$.

2) прочесс $\left(X_{t \wedge \tau_{n}}, \mathscr{F}_{t}, \widetilde{\mathbf{Q}}^{n}\right)_{t \in[0, T]}$ является семимартингалом с разложением

$$
\begin{aligned}
X_{t \wedge \tau_{n}}=X_{0}+\int_{0}^{t} & I_{\left\{s \leqslant \tau_{n}\right\}}\left[a_{s}(X)+b_{s}(X) \sigma_{s}(X)\right. \\
& \left.+\int_{\mathbf{R}} h_{s}(X, z) \varphi_{s}(X, z) K(d z)\right] d s+\widetilde{\mathscr{M}}_{t}^{c, n}+\widetilde{\mathscr{M}}_{t}^{d, n},
\end{aligned}
$$

в котором $\left(\widetilde{\mathscr{M}}_{t}^{c, n} ; \mathscr{F}_{t}, \mathbf{Q}^{n}\right)_{t \in[0, T]} u\left(\widetilde{\mathscr{M}}_{t}^{d, n} ; \mathscr{F}_{t}, \mathbf{Q}^{n}\right)_{t \in[0, T]}-$ непрерывныици и чисто разрьвный квадратично интегрируемье мартингаль. Их предсказуемье квадратические характеристики задаются соответственно формулами:

$$
\begin{aligned}
\left\langle\widetilde{\mathscr{M}}^{c, n}\right\rangle_{t} & =\int_{0}^{t} I_{\left\{s \leqslant \tau_{n}\right\}} b_{s}^{2}(X) d s, \\
\left\langle\widetilde{\mathscr{M}}^{d, n}\right\rangle_{t} & =\int_{0}^{t} \int_{\mathbf{R}} I_{\left\{s \leqslant \tau_{n}\right\}} h_{s}^{2}(X, z)\left[1+\varphi_{s}(X, z)\right] K(d z) d s .
\end{aligned}
$$

Д о к а з а т е л ь с т в о. 1) Пусть $\theta_{n}-$ момент остановки, $\theta_{n} \leqslant \tau_{n}$. Пусть $\Gamma \in \mathbf{R} \backslash\{0\}$ - измеримое множество. Тогда

$$
\widetilde{\mathbf{E}}^{n} \int_{0}^{T} \int_{\mathbf{R}} I_{\left\{s \leqslant \theta_{n}\right\}} I_{\{z \in \Gamma\}} \mu(d s, d z)=\widetilde{\mathbf{E}}^{n} \int_{0}^{T} \int_{\mathbf{R}} I_{\left\{s \leqslant \theta_{n}\right\}} I_{\{z \in \Gamma\}} \nu^{n}(d s, d z) .
$$


С другой стороны,

$$
\begin{aligned}
\widetilde{\mathbf{E}}^{n} & \int_{0}^{T} \int_{\mathbf{R}} I_{\left\{s \leqslant \theta_{n}\right\}} I_{\{z \in \Gamma\}} \mu(d s, d z)=\mathbf{E}_{\mathfrak{z}_{T \wedge \theta_{n}}} \int_{0}^{T} \int_{\mathbf{R}} I_{\left\{s \leqslant \theta_{n}\right\}} I_{\{z \in \Gamma\}} \mu^{n}(d s, d z) \\
& =\mathbf{E} \int_{0}^{T} \int_{\mathbf{R}} I_{\left\{s \leqslant \theta_{n}\right\}} I_{\{z \in \Gamma\} \mathfrak{z}_{s \wedge \theta_{n}}} \mu^{n}(d s, d z) \\
& =\mathbf{E} \int_{0}^{T} \int_{\mathbf{R}} I_{\left\{s \leqslant \theta_{n}\right\}} I_{\{z \in \Gamma\} \mathfrak{z}_{\left(s \wedge \theta_{n}\right)-}}\left[1+\varphi_{s}(X, z)\right] d s K(d z) \\
& =\mathbf{E}_{\mathfrak{z}_{T \wedge \theta_{n}}} \int_{0}^{T} \int_{\mathbf{R}} I_{\left\{s \leqslant \theta_{n}\right\}} I_{\{z \in \Gamma\}}\left[1+\varphi\left(s, X_{s-}, z\right)\right] d s K(d z) \\
& =\widetilde{\mathbf{E}}^{n} \int_{0}^{T} \int_{\mathbf{R}} I_{\left\{s \leqslant \theta_{n}\right\}} I_{\{z \in \Gamma\}}\left[1+\varphi\left(s, X_{s-}, z\right)\right] d s K(d z) .
\end{aligned}
$$

Следовательно, в силу произвольности $\theta_{n}$ и $\Gamma$, утверждение 1$)$ имеет место (по поводу дополнительных деталей см. $[25, \S 5$, гл. 4] и $[13, \S 3 . \mathrm{a}-$ $\S 3 . d])$.

2) Для удобства обозначений положим

$$
\begin{gathered}
\mathscr{M}_{t}^{c, n}=\int_{0}^{t} I_{\left\{s \leqslant \tau_{n}\right\}} b_{s}(X) d B_{s}, \\
\mathscr{M}_{t}^{d, n}=\int_{0}^{t} \int_{\mathbf{R}} I_{\left\{s \leqslant \tau_{n}\right\}} h_{s}(X, z)[\mu(d s, d z)-d s K(d z)] .
\end{gathered}
$$

Поскольку $\mathfrak{z}_{t \wedge \tau_{n}}$ и $\mathscr{M}_{t}^{c, n}, \mathscr{M}_{t}^{d, n}-$ квадратично интегрируемые мартингалы, совместные предсказуемая квадратическая характеристика $\left.\left\langle\mathfrak{z} \cdot \wedge \tau_{n}, \mathscr{M}^{c, n}\right\rangle_{t}=\int_{0}^{t} I_{\left\{s \leqslant \tau_{n}\right\}} \mathfrak{z}_{s-} \sigma_{s}(X) b_{s}(X)\right) d s$ и квадратическая характеристика

$$
\left.\left[\mathfrak{z} \cdot \wedge \tau_{n}, \mathscr{M}^{d, n}\right\rangle\right]_{t}=\int_{0}^{t} \int_{\mathbf{R}} I_{\left\{s \leqslant \tau_{n}\right\}} \mathfrak{z} s-_{s-}(X, z) h_{s}(X, z) \mu(d s, d z)
$$

полностью определены. Более того, $\left\langle\mathfrak{z} \cdot \wedge \tau_{n}, \mathscr{M}^{d, n}\right\rangle_{t}$, являясь компенсатором квадратической характеристики $\left.\left[\mathfrak{z}_{\cdot} \wedge \tau_{n}, \mathscr{M}^{d, n}\right\rangle\right]_{t}$, допускает следующее представление:

$$
\left\langle\mathfrak{z} \cdot \wedge \tau_{n} \mathscr{M}^{d, n}\right\rangle_{s}=\int_{0}^{t} \int_{\mathbf{R}} I_{\left\{s \leqslant \tau_{n}\right\}} \mathfrak{z}_{s-} \varphi_{s}(X, z) h_{s}(X, z) K(d z) d s .
$$

Далее, в силу теоремы 2 из $[25, \S 5$, гл. 4], процессы

$$
\begin{aligned}
& \widetilde{\mathscr{M}}_{t}^{c, n}=\mathscr{M}_{t}^{c, n}-\int_{0}^{t} \mathfrak{z}_{\left(s \wedge \tau_{n}\right)-}^{-1} d\left\langle\mathfrak{z}_{\cdot} \wedge \tau_{n} \mathscr{M}^{c, n}\right\rangle_{s}, \\
& \widetilde{\mathscr{M}}_{t}^{d, n}=\mathscr{M}_{t}^{d, n}-\int_{0}^{t} \mathfrak{z}_{\left(s \wedge \tau_{n}\right)-}^{-1} d\left\langle\mathfrak{z} \cdot \wedge \tau_{n}, \mathscr{M}^{d, n}\right\rangle_{s}
\end{aligned}
$$


являются непрерывным и чисто разрывным $\mathbf{Q}^{n}$-мартингалами. Другими словами, $\left(\mathbf{Q}^{n}: \mathscr{M}_{t}^{c, n} ; \mathscr{M}_{t}^{d, n}\right)$-процессы определяются следующим образом:

$$
\begin{aligned}
\mathscr{M}_{t}^{c, n} & =\int_{0}^{t} I_{\left\{s \leqslant \tau_{n}\right\}} \sigma_{s}(X) b_{s}(X) d s+\widetilde{\mathscr{M}}_{t}^{c, n} \\
\mathscr{M}_{t}^{d, n} & =\int_{0}^{t} \int_{\mathbf{R}} I_{\left\{s \leqslant \tau_{n}\right\}} \varphi_{s}(X, z) h_{s}(X, z) d s+\widetilde{\mathscr{M}}_{t}^{d, n}
\end{aligned}
$$

где $\left(\mathbf{Q}^{n}, \widetilde{\mathscr{M}}_{t}^{c, n}\right)$ — непрерывный мартингал и $\left(\mathbf{Q}^{n}, \widetilde{\mathscr{M}}_{t}^{d, n}\right)$ - чисто разрывный мартингал с $\left\langle\mathscr{\mathscr { M }}^{c, n}\right\rangle_{t}$ и $\left\langle\widetilde{\mathscr{M}}^{d, n}\right\rangle_{t}$, определенными в (А.1).

\section{СПИСОК ЛИТЕРАТУРЫ}

1. Andersen L. B., Piterbarg V. V. Moment explosions in stochastic volatility models. Finance Stoch., 2007, v. 11, p. 29-50.

2. Baudoin F., Nualart D. Equivalence of Volterra processes. - Stochastic Process. Appl., 2003, v. 107, p. 327-350.

3. Benes V. E. Existence of optimal stochastic control laws. - SIAM J. Control, 1971, v. 9 , p. $446-475$.

4. Cheridito P. Mixed fractional Brownian motion. - Bernoulli, 2001, v. 7, p. 913-934.

5. Cheridito P., Filipović D., Yor M. Equivalent and absolutely continuous measure changes for jump-diffusion processes. - Ann. Appl. Probab., 2005, v. 15, № 3, p. $1713-1732$.

6. Cox J. C. The constant elasticity of variance option. Pricing model. - J. Portfolio Management, 1997, v. 23, № 2, p. 15-17.

7. Dawson D. Equivalence of Markov processes. - Trans. Amer. Math. Soc., 1968, v. 131, p. $1-31$.

8. Delbaen F., Shirakawa $H$. A note on option pricing for constant elasticity of variance model. - Asia-Pac. Financ. Mark., 2002, v. 9, № 2, p. 85-99.

9. Doléans-Dade C. Quelques applications de la formule de changement de variables pour les semi-martingales. - Z. Wahrscheinlichkeitstheor. verw. Geb., 1970, v. 16, p. $181-194$.

10. Гирсанов И. В. О преобразовании некоторого класса случайных процессов с помощью абсолютно непрерывной замены меры. - Теория вероятн. и ее примен., 1960 , т. 5, в. 2, с. 285-301.

11. Hitsuda $M$. Representation of Gaussian processes equivalent to Wiener process. Osaka J. Math., 1968, v. 5, p. 299-312.

12. Itô K., Watanabe $S$. Transformation of Markov processes by multiplicative functionals. - Ann. Inst. Fourier (Grenoble), 1965, v. 15, p. 13-30.

13. Жакод Ж., Ширяев А. Н. Предельные теоремы для случайных процессов, т. 1, 2. М.: Наука, 1994, 542 с., 366 с.

14. Кабанов Ю.М., Липцер Р.Ш., Ширяев А.Н. Абсолютная непрерывность и сингулярность локально абсолютно непрерывных вероятностных распределений. I, II. - Матем. сб., 1978, т. 107(149), № 3(11), с. 364-415; 1979, т. 108(150), № 1 , c. $32-61$.

15. Kadota T., Shepp L. Conditions for absolute continuity between a certain pair of probability measures. - Z. Wahrscheinlichkeitstheor. verw. Geb., 1970, v. 16, p. 250260.

16. Kallsen J., Shiryaev A. N. The cumulant process and Esschers change of measure. Finance Stoch., 2002, v. 6, p. 397-428. 
17. Karatzas I., Shreve S.E. Brownian Motion and Stochastic Calculus. New YorkBerlin-Heidelberg: Springer-Verlag, 1991, 470 p. (Graduate Texts in Math., v. 113.)

18. Kazamaki N. On a problem of Girsanov. - Tôhoku Math. J., 1970, v. 29, p. 597-600.

19. Kazamaki N., Sekiguchi T. On the transformation of some classes of martingales by a change of law. - Tôhoku Math. J., 1979, v. 31, p. 261-279.

20. Krylov N. V. A simple proof of a result of A. Novikov. arXiv:math/020713v2 [math.PR], 2009.

21. Kunita H. Absolute continuity of Markov processes and generators. - Nagoya Math. J., 1969, v. 36, p. 1-26.

22. Kunita H. Absolute continuity of Markov processes. - Lecture Notes in Math., 1976, v. 511, p. 44-77.

23. Lépingle D., Mémin J. Sur lintégrabilité uniforme des martingales exponentielles. Z. Wahrscheinlichkeitstheor. verw. Geb., 1978, v. 42, p. 175-203.

24. Липцер Р.Ш., Ширяев А.Н. Об абсолютной непрерывности мер, соответствующих процессам диффузионного типа, относительно винеровской. - Изв. АН CCCP, 1972 , т. 36, № 4 , с. 847-889.

25. Липчер Р. Ш., Ширяев А.Н. Теория мартингалов. М.: Наука, 1986, 512 с.

26. Liptser R.S., Shiryaev A.N. Statistics of Random Processes. I: General Theory. Berlin: Springer, 2001, 427 p. (Appl. Math., v. 5.)

27. Liptser R.S., Shiryaev A.N. Statistics of Random Processes II: Applications. Berlin: Springer, 2001, 402 p. (Appl. Math., v. 6.)

28. Liptser $R$. Benês condition for discontinuous exponential martingale. arXiv: 0911.0641v3 [math.PR]

29. Mijatović A., Urusov M. On the martingale property of certain local martingales: criteria and applications. - arXiv:0905.3701v1 [math.PR]; Probab. Theory Related Fields, 2012, v. 152, № 1-2, p. 1-30.

30. Новиков A. А. Об условиях равномерной интегрируемости непрерывных неотрицательных мартингалов. - Теория вероятн. и ее примен., 1979, т. 24, № 4, с. 821825.

31. Palmowski Z., Rolski T. A technique for exponential change of measure for Markov processes. - Bernoulli, 2002, v. 8, p. 767-785.

32. Revuz D., Yor M. Continuous Martingales and Brownian Moton. Berlin: SpringerVerlag, 1991, 533 p. (Grundlehren Math. Wiss., v. 293.)

33. Rydberg T. A note on the existence of equivalent martingale measures in a Markovian setting. - Finance Stoch., 1997, v. 1, p. 251-257.

34. Picard J. Representation formulae for the fractional Brownian motion. - Lecture Notes in Math., 2011, v. 2006, p. 3-70; arXiv:0912.3168v2 [math.PR]

35. Ширяев А. Н. Основы стохастической финансовой математики. Т. 1: Факты. Модели. Т. 2: Теория. М.: Фазис, 2004, 1017 с.

36. Sin C. A. Complications with stochastic volatility models. - Adv. Appl. Probab., 1998 , v. 30 , № 1 , p. $256-268$.

37. Wong B., Heyde C. C. On the martingale property of stochastic exponentials. - J. Appl. Probab., 2004, v. 41, p. 654-664. 